\title{
Passivity of Memristive BAM Neural Networks with Probabilistic and Mixed Time-Varying Delays
}

\author{
Weiping Wang $\mathbb{D},{ }^{1,2}$ Meiqi Wang, ${ }^{1,2}$ Xiong Luo $\mathbb{D}^{1,2}$ Lixiang $L i \mathbb{D}^{3},{ }^{3}$ and Wenbing Zhao $\mathbb{D}^{4}$ \\ ${ }^{1}$ School of Computer and Communication Engineering, University of Science and Technology Beijing, Beijing 100083, China \\ ${ }^{2}$ Beijing Key Laboratory of Knowledge Engineering for Materials Science, Beijing 100083, China \\ ${ }^{3}$ Information Security Center, State Key Laboratory of Networking and Switching Technology, \\ Beijing University of Posts and Telecommunications, Beijing 100876, China \\ ${ }^{4}$ Department of Electrical Engineering and Computer Science, Cleveland State University, Cleveland, OH 44115, USA
}

Correspondence should be addressed to Weiping Wang; shiya666888@126.com and Xiong Luo; xluo@ustb.edu.cn

Received 20 June 2017; Revised 17 November 2017; Accepted 21 January 2018; Published 1 April 2018

Academic Editor: Renming Yang

Copyright (C) 2018 Weiping Wang et al. This is an open access article distributed under the Creative Commons Attribution License, which permits unrestricted use, distribution, and reproduction in any medium, provided the original work is properly cited.

This paper is concerned with the passivity problem of memristive bidirectional associative memory neural networks (MBAMNNs) with probabilistic and mixed time-varying delays. By applying random variables with Bernoulli distribution, the information of probability time-varying delays is taken into account. Furthermore, we consider the probability distribution of the variation and the extent of the delays; therefore, the results derived are less conservative than in the existing papers. In particular, the leakage delays as well as distributed delays are all taken into consideration. Based on appropriate Lyapunov-Krasovskii functionals (LKFs) and some useful inequalities, several conditions for passive performance are established in linear matrix inequalities (LMIs). Finally, numerical examples are given to demonstrate the feasibility of the presented theories, and the results reveal that the probabilistic and mixed time-varying delays have an unstable influence on the system and should not be ignored.

\section{Introduction}

Bidirectional associative memory neural networks (BAMNNs) are a class of two-layer neural systems, which were first introduced by Kosko in 1987. The neurons in the first layer are connected to another layer, and in the same layer, the neurons are not interconnected [1-3]. Owing to their special structure, BAMNNs have displayed many good features in various areas such as signal processing, image processing, and optimization problems [4-6]. In 2015, the stability of inertial BAMNNs with time-varying delay via impulsive control was discussed in [7]. Zhang et al. considered the exponential stability of BAMNNs with time-varying delays in [8]. Wang et al. addressed the global asymptotic stability of impulsive fractional-order BAMNNs with time delay in [9].

Memristor, a combination of a resistor and memory, has received increasing attention in many fields [10-13]. By applying the nonvolatile feature of the memristor, researchers were able to develop MBAMNN models. Because of the pinched hysteresis effects, MBAMNNs have a memory function, which can be used to imitate the human brain [14, 15]. In 2015, nonfragile synchronization of MBAMNNs with random feedback gain fluctuations was investigated in [16]. Based on functional differential inclusions, Jiang et al. obtained the dynamic behaviors for MBAMNNs with timevarying delays in [17].

Moreover, passivity is a special case of a broader theory of dissipativity, which plays a significant role in the stability analysis of dynamical systems, nonlinear control, and other areas. The main innate character of passivity theory is that the passive characteristics can make the system internally stable [18-20]. In recent years, many researchers have proposed passivity analysis for memristive neural networks (MNNs). Liu and $\mathrm{Xu}$ investigated the passivity analysis of MNNs with different state-dependent memductance functions and mixed time-varying delays in [21]. In 2016, the passivity of MBAMNNs with uncertain delays and different memductance was investigated in [22]. Nevertheless, there are 
few people to study the passivity of MBAMNNs, which encourages our idea.

In the human brain, the transmission of information in neurons is often accompanied by a time delay, so time delay is inevitable in the neural networks, which is the origin of oscillation, divergence, and so forth [23-33]. Sometimes, the value of delay may be very large, but the probability of such delay is very small. Therefore, we use the probability distribution of time delay in the interval to reflect an actual situation better. Furthermore, it is clearer to describe the probabilistic time-varying delays through introducing random variables with Bernoulli distribution. In recent years, some researchers have discussed the probabilistic time-varying delays in the neural networks $[34,35]$. In 2016, Pradeep et al. investigated the robust stability analysis of stochastic neural networks with probabilistic time-varying delays in [36]. Li et al. considered passivity analysis of memristive neural networks with probabilistic time-varying delays in [37]. Hence, it is of great importance to research the passivity of MBAMNNs with probabilistic time-varying delays.

In addition, there also exist two types of time-varying delays named leakage delays (or forgetting delays) and distributed time delays. The research of leakage time delay can be traced back to the early 90 s of the last century; researchers found out that, due to the delay in switching time or signal transmission, there is a time delay in the negative feedback term of the network system; this delay is named leakage time delay. As is well known, leakage delays exist in many real systems such as population dynamics and neural networks [38, 39]. Moreover, leakage delay also has a significant influence on the dynamics of neural networks because it has been shown that such kind of time delay in the leakage term has a tendency to destabilize a system. Under the influence of leakage and additive time-varying delays, robust passivity analysis for neural networks was addressed in [40]. In 2016, the robust stability analysis for discrete-time neural networks with leakage delays was studied in [41].

On the other hand, due to the presence of multiple parallel paths with a variety of neuronal synapses' lengths and sizes, there is a spatial width of the network, and then there may exist either a distribution of the transmission voltage in these parallel paths or a distribution of transmission delays over a period of time. Hence, the distribution delay is used to describe this phenomenon $[42,43]$. In 2015, Du et al. investigated the passivity of neural networks with discrete and distributed time-varying delays in [44]. In 2016, Yang et al. considered finite-time stabilization of uncertain neural networks with distributed time-varying delays in [45]. However, to the best of our knowledge, there are few results on the passivity of MBAMNNs with probabilistic, leakage, and distributed time-varying delays. Thus, it is significant to study the passivity of MBAMNNs with these time-varying delays.

Motivated by the main points discussed above, the contribution of this paper lies in three aspects.

(1) This is the first attempt to discuss the passivity analysis of MBAMNNs with probabilistic and mixed time-varying delays. In particular, the leakage delays as well as distributed delays are all taken into consideration.
(2) The LKFs that we designed include double and triple integral terms, and by applying some helpful inequalities, the passivity analysis of MBAMNNs becomes less conservative than the existing results $[19,21]$.

(3) After using MATLAB LMI control toolbox, all the derived results are expressed in LMIs, and a feasible solution can be easily obtained.

The rest of the paper is structured as follows. In Section 2, we introduce the model of the MBAMNNs with probabilistic time-varying delays. In Section 3, the main results on passivity analysis of MBAMNNs with probabilistic and mixed time-varying delays are derived. In Section 4, some numerical simulations are provided to demonstrate the feasibility of our results. In Section 5, the conclusion is shown.

\section{Model Description and Preliminaries}

In this paper, we propose the MBAMNN with probabilistic time-varying delays as follows:

$$
\begin{aligned}
& \dot{x}_{i}(t)=-C_{i} x_{i}(t)+\sum_{j=1}^{m} a_{j i}\left(x_{i}(t)\right) f_{j}\left(y_{j}(t)\right) \\
&+\sum_{j=1}^{m} b_{j i}\left(x_{i}(t)\right) f_{j}\left(y_{j}(t-\tau(t))\right)+u_{i}(t), \\
& \dot{y}_{j}(t)=-D_{j} y_{j}(t)+\sum_{i=1}^{n} e_{i j}\left(y_{j}(t)\right) g_{i}\left(x_{i}(t)\right) \\
&+\sum_{i=1}^{n} h_{i j}\left(y_{j}(t)\right) g_{i}\left(x_{i}(t-d(t))\right)+v_{j}(t), \\
& i=1, \ldots, n, j=1, \ldots, m,
\end{aligned}
$$

or it can be rewritten as follows:

$$
\begin{aligned}
\dot{x}(t)= & -C x(t)+A(x(t)) f(y(t)) \\
& +B(x(t)) f(y(t-\tau(t)))+u(t), \\
\dot{y}(t)= & -D y(t)+E(y(t)) g(x(t)) \\
& +H(y(t)) g(x(t-d(t)))+v(t),
\end{aligned}
$$

where $x_{i}(t)$ and $y_{j}(t)$ denote the state variables related to the $i$ th and $j$ th neurons. $A(x(t))=\left(a_{j i}\left(x_{i}(t)\right)\right)_{m \times n}$ and $E(y(t))=\left(e_{i j}\left(y_{j}(t)\right)\right)_{n \times m}$ are the connection weight matrices, $f_{j}(\cdot)$ and $g_{i}(\cdot)$ are the activation functions, and $B(x(t))=\left(b_{j i}\left(x_{i}(t)\right)\right)_{m \times n}$ and $H(y(t))=\left(h_{i j}\left(y_{j}(t)\right)\right)_{n \times m}$ are the delayed connection weight matrices. The self-feedback connection weights $C_{i}$ and $D_{j}$ are positive diagonal matrices. $u_{i}(t)$ and $v_{j}(t)$ represent the continuous external inputs; the nonnegative continuous variables $\tau(t)$ and $d(t)$ correspond to the time-varying delays.

Assumption 1. The functions $f_{j}(t)(j=1,2, \ldots, m)$ and $g_{i}(t)(i=1,2, \ldots, n)$ are bounded and continuous and satisfy the conditions as follows: 


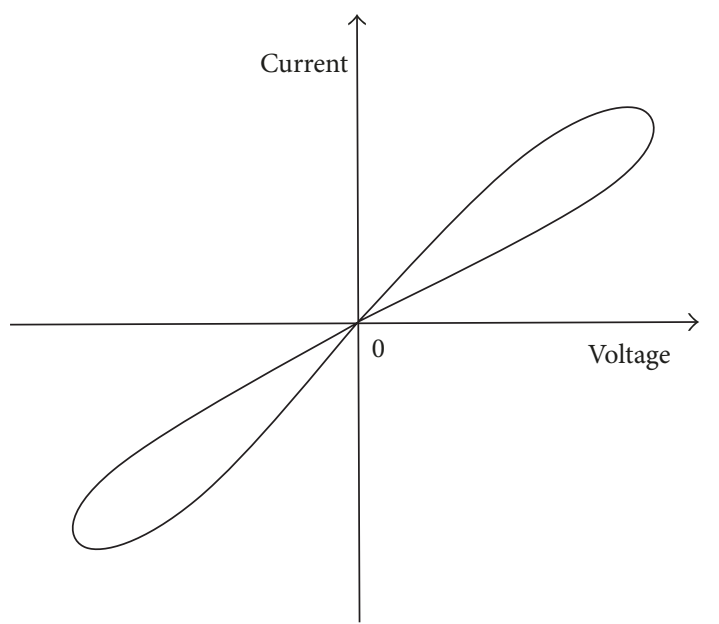

FIGURE 1: The typical current-voltage characteristic of a memristor.

$$
\begin{gathered}
\varsigma_{j}^{-} \leq \frac{f_{j}(b)-f_{j}(a)}{b-a} \leq \varsigma_{j}^{+}, \\
\sigma_{i}^{-} \leq \frac{g_{i}(b)-g_{i}(a)}{b-a} \leq \sigma_{i}^{+},
\end{gathered}
$$

with $\varsigma^{-}=\operatorname{diag}\left(\varsigma_{1}^{-}, \varsigma_{2}^{-}, \ldots, \varsigma_{m}^{-}\right), \varsigma^{+}=\operatorname{diag}\left(\varsigma_{1}^{+}, \varsigma_{2}^{+}, \ldots, \varsigma_{m}^{+}\right), \sigma^{-}=$ $\operatorname{diag}\left(\sigma_{1}^{-}, \sigma_{2}^{-}, \ldots, \sigma_{n}^{-}\right)$, and $\sigma^{+}=\operatorname{diag}\left(\sigma_{1}^{+}, \sigma_{2}^{+}, \ldots, \sigma_{n}^{+}\right), a, b \in$ $R, a \neq b$.

Based on the current-voltage characteristic and the feature of memristor, the memristive connection weights $a_{j i}\left(x_{i}(t)\right), b_{j i}\left(x_{i}(t)\right), e_{i j}\left(y_{j}(t)\right)$, and $h_{j i}\left(x_{i}(t)\right)$ will change with time. Then, we let

$$
\begin{array}{r}
a_{j i}\left(x_{i}(t)\right)= \begin{cases}\widehat{a}_{j i}, & \left|x_{i}(t)\right|>\Theta_{i}, \\
\check{a}_{j i}, & \left|x_{i}(t)\right| \leq \Theta_{i},\end{cases} \\
b_{j i}\left(x_{i}(t)\right)= \begin{cases}\widehat{b}_{j i}, & \left|x_{i}(t)\right|>\Theta_{i}, \\
\check{b}_{j i}, & \left|x_{i}(t)\right| \leq \Theta_{i},\end{cases} \\
e_{i j}\left(y_{j}(t)\right)= \begin{cases}\widehat{e}_{i j}, & \left|y_{j}(t)\right|>\Psi_{j}, \\
\check{e}_{i j}, & \left|y_{j}(t)\right| \leq \Psi_{j},\end{cases} \\
h_{i j}\left(y_{j}(t)\right)= \begin{cases}\widehat{f}_{i j}, & \left|y_{j}(t)\right|>\Psi_{j}, \\
\check{f}_{i j}, & \left|y_{j}(t)\right| \leq \Psi_{j},\end{cases}
\end{array}
$$

in which $a_{j i}, b_{j i}, e_{i j}$, and $h_{i j}$ are constants and the switching jumps $\Theta_{i}>0, \Psi_{j}>0$.

Based on Figures 1 and 2, it is clear that $a_{j i}\left(x_{i}(t)\right)$, $b_{j i}\left(x_{i}(t)\right), e_{i j}\left(y_{j}(t)\right)$, and $h_{i j}\left(y_{j}(t)\right)$ are piecewise continuous functions; the solutions of the systems are indicated in Filippov's sense and the interval is represented by $[\cdot, \cdot]$. Set

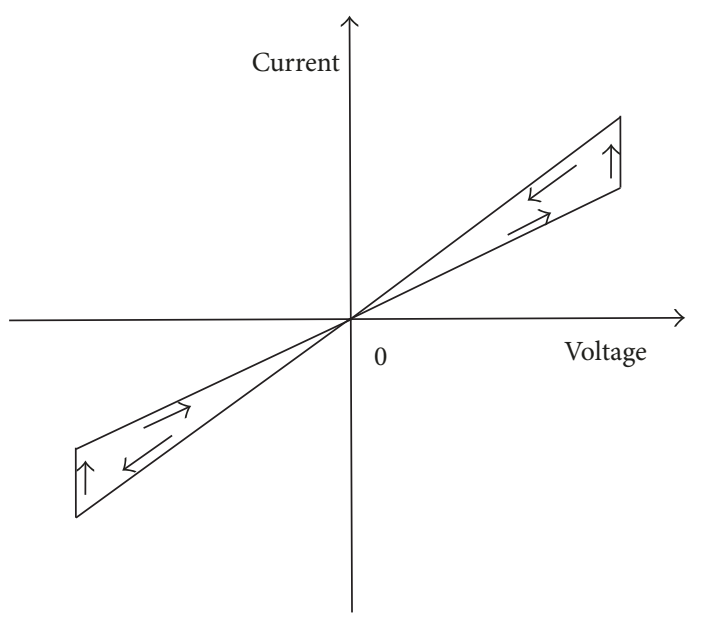

FIGURE 2: The characteristic of a piecewise linear charge-controlled memristor.

$$
\begin{aligned}
& \bar{a}_{j i}=\max \left\{\widehat{a}_{j i}, \check{a}_{j i}\right\}, \\
& \underline{a}_{j i}=\min \left\{\widehat{a}_{j i}, \check{a}_{j i}\right\}, \\
& \bar{b}_{j i}=\max \left\{\widehat{b}_{j i}, \check{b}_{j i}\right\}, \\
& \underline{b}_{j i}=\min \left\{\widehat{b}_{j i}, \check{b}_{j i}\right\}, \\
& \bar{e}_{i j}=\max \left\{\widehat{e}_{i j}, \check{e}_{i j}\right\}, \\
& \underline{e}_{i j}=\min \left\{\widehat{e}_{i j}, \check{e}_{i j}\right\}, \\
& \bar{h}_{i j}=\max \left\{\widehat{h}_{i j}, \check{h}_{i j}\right\}, \\
& \underline{h}_{i j}=\min \left\{\widehat{h}_{i j}, \check{h}_{i j}\right\},
\end{aligned}
$$

for $i=1,2, \ldots, n, j=1,2, \ldots, m$. $\operatorname{co}[\bar{r}, \underline{r}]$ indicates the convex closure of $[\bar{r}, \underline{r}]$. Obviously, the set-valued maps are defined as

$$
\begin{aligned}
& \operatorname{co}\left[a_{j i}\left(x_{i}(t)\right)\right]= \begin{cases}\widehat{a}_{j i}, & \left|x_{i}(t)\right|>\Theta_{i}, \\
{\left[\underline{a}_{j i}, \bar{a}_{j i}\right],} & \left|x_{i}(t)\right|=\Theta_{i}, \\
\check{a}_{j i}, & \left|x_{i}(t)\right|<\Theta_{i},\end{cases} \\
& \operatorname{co}\left[b_{j i}\left(x_{i}(t)\right)\right]= \begin{cases}\widehat{b}_{j i}, & \left|x_{i}(t)\right|>\Theta_{i}, \\
{\left[\underline{b}_{j i}, \bar{b}_{j i}\right],} & \left|x_{i}(t)\right|=\Theta_{i}, \\
\check{b}_{j i}, & \left|x_{i}(t)\right|<\Theta_{i},\end{cases} \\
& \operatorname{co}\left[e_{i j}\left(y_{j}(t)\right)\right]= \begin{cases}\widehat{e}_{i j}, & \left|y_{j}(t)\right|>\Psi_{j}, \\
{\left[\underline{e}_{i j}, \bar{e}_{i j}\right],} & \left|y_{j}(t)\right|=\Psi_{j}, \\
\check{e}_{i j}, & \left|y_{j}(t)\right|<\Psi_{j},\end{cases}
\end{aligned}
$$




$$
\operatorname{co}\left[h_{i j}\left(y_{j}(t)\right)\right]= \begin{cases}\widehat{h}_{i j}, & \left|y_{j}(t)\right|>\Psi_{j}, \\ {\left[\underline{h}_{i j}, \bar{h}_{i j}\right],} & \left|y_{j}(t)\right|=\Psi_{j}, \\ \check{h}_{i j}, & \left|y_{j}(t)\right|<\Psi_{j} .\end{cases}
$$

As a matter of convenience, we make the following assumptions.

Assumption 2. We define the probability distribution of time delays $\tau(t)$ and $d(t)$ as follows:

$$
\begin{gathered}
\operatorname{Prob}\left\{\tau(t) \in\left[0, \tau_{1}\right]\right\}=\mu_{0}, \\
\operatorname{Prob}\left\{\tau(t) \in\left[\tau_{1}, \tau_{2}\right]\right\}=1-\mu_{0}, \\
\operatorname{Prob}\left\{d(t) \in\left[0, d_{1}\right]\right\}=\omega_{0}, \\
\operatorname{Prob}\left\{d(t) \in\left[d_{1}, d_{2}\right]\right\}=1-\omega_{0},
\end{gathered}
$$

where $0 \leq \mu_{0} \leq 1$ and $0 \leq \omega_{0} \leq 1$ are constants.

Thus, the random variables $\mu(t)$ and $\omega(t)$ can be defined as

$$
\begin{aligned}
& \mu(t)= \begin{cases}1, & \tau(t) \in\left[0, \tau_{1}\right], \\
0, & \tau(t) \in\left[\tau_{1}, \tau_{2}\right],\end{cases} \\
& \omega(t)= \begin{cases}1, & d(t) \in\left[0, d_{1}\right], \\
0, & d(t) \in\left[d_{1}, d_{2}\right] .\end{cases}
\end{aligned}
$$

Then, it can be derived that $\mu(t)$ and $\omega(t)$ are Bernoulli distributed sequences with

$$
\begin{aligned}
& \operatorname{Prob}\{\mu(t)=1\}=\mu_{0}, \\
& \operatorname{Prob}\{\mu(t)=0\}=1-\mu_{0}, \\
& \operatorname{Prob}\{\omega(t)=1\}=\omega_{0}, \\
& \operatorname{Prob}\{\omega(t)=0\}=1-\omega_{0} .
\end{aligned}
$$

According to Assumption 2, it is easy to see that

$$
\begin{gathered}
\mathbb{E}\left\{\mu(t)-\mu_{0}\right\}=0, \\
\mathbb{E}\left\{\left(\mu(t)-\mu_{0}\right)^{2}\right\}=\mu_{0}\left(1-\mu_{0}\right), \\
\mathbb{E}\left\{\omega(t)-\omega_{0}\right\}=0, \\
\mathbb{E}\left\{\left(\omega(t)-\omega_{0}\right)^{2}\right\}=\omega_{0}\left(1-\omega_{0}\right) .
\end{gathered}
$$

Now, time-varying delays $\tau_{1}(t), \tau_{2}(t), d_{1}(t)$, and $d_{2}(t)$ are introduced as

$$
\begin{gathered}
\tau(t)= \begin{cases}\tau_{1}(t), & \tau(t) \in\left[0, \tau_{1}\right], \\
\tau_{2}(t), & \tau(t) \in\left[\tau_{1}, \tau_{2}\right],\end{cases} \\
d(t)= \begin{cases}d_{1}(t), & d(t) \in\left[0, d_{1}\right], \\
d_{2}(t), & d(t) \in\left[d_{1}, d_{2}\right] .\end{cases}
\end{gathered}
$$

Assumption 3. Here, constants $\tau_{1}, \tau_{2}, d_{1}, d_{2}, \mu_{1}, \mu_{2}, \omega_{1}$, and $\omega_{2}$ exist, such that

$$
\begin{aligned}
0 & \leq \tau_{1}(t) \leq \tau_{1}, \\
\dot{\tau}_{1}(t) & \leq \mu_{1}, \\
0 & \leq \tau_{2}(t) \leq \tau_{2}, \\
\dot{\tau}_{2}(t) & \leq \mu_{2}, \\
0 & \leq d_{1}(t) \leq d_{1}, \\
\dot{d}_{1}(t) & \leq \omega_{1}, \\
0 & \leq d_{2}(t) \leq d_{2}, \\
\dot{d}_{2}(t) & \leq \omega_{2} .
\end{aligned}
$$

Assumption 4. The leakage delays $\delta(t)$ and $\rho(t)$ and distributed delays $\alpha(t)$ and $\beta(t)$ satisfy

$$
\begin{aligned}
0 & \leq \delta(t) \leq \delta, \\
\dot{\delta}(t) & \leq \delta_{0}, \\
0 & \leq \rho(t) \leq \rho, \\
\dot{\rho}(t) & \leq \rho_{0}, \\
0 & \leq \alpha(t) \leq \alpha, \\
0 & \leq \beta(t) \leq \beta .
\end{aligned}
$$

By employing the theories of set-valued maps, differential inclusions, stochastic variables $\mu_{0}, \omega_{0}$, and new functions $\tau_{1}(t), \tau_{2}(t), d_{1}(t)$, and $d_{2}(t)$, system (1) becomes

$$
\begin{aligned}
\dot{x}_{i}(t) & \\
\epsilon & -C_{i} x_{i}(t)+\sum_{j=1}^{m} \mathrm{co}\left[a_{j i}\left(x_{i}(t)\right)\right] f_{j}\left(y_{j}(t)\right) \\
& +\mu(t) \sum_{j=1}^{m} \operatorname{co}\left[b_{j i}\left(x_{i}(t)\right)\right] f_{j}\left(y_{j}\left(t-\tau_{1}(t)\right)\right) \\
& +(1-\mu(t)) \sum_{j=1}^{m} \operatorname{co}\left[b_{j i}\left(x_{i}(t)\right)\right] f_{j}\left(y_{j}\left(t-\tau_{2}(t)\right)\right) \\
& +u_{i}(t), \\
\dot{y}_{j}(t) & \\
\epsilon & -D_{j} y_{j}(t)+\sum_{i=1}^{n} \mathrm{co}\left[e_{i j}\left(y_{j}(t)\right)\right] g_{i}\left(x_{i}(t)\right) \\
& +\omega(t) \sum_{i=1}^{n} \cos \left[h_{i j}\left(y_{j}(t)\right)\right] g_{i}\left(x_{i}\left(t-d_{1}(t)\right)\right) \\
& +(1-\omega(t)) \sum_{i=1}^{n} \operatorname{co}\left[h_{i j}\left(y_{j}(t)\right)\right] g_{i}\left(x_{i}\left(t-d_{2}(t)\right)\right)
\end{aligned}
$$


Or it can be rewritten as follows:

$$
\begin{aligned}
\dot{x}(t)= & -C x(t)+A(x(t)) f(y(t)) \\
& +\mu(t) B(x(t)) f\left(y\left(t-\tau_{1}(t)\right)\right) \\
& +(1-\mu(t)) B(x(t)) f\left(y\left(t-\tau_{2}(t)\right)\right) \\
& +u(t), \\
\dot{y}(t)= & -D y(t)+E(y(t)) g(x(t)) \\
& +\omega(t) H(y(t)) g\left(x\left(t-d_{1}(t)\right)\right) \\
& +(1-\omega(t)) H(y(t)) g\left(x\left(t-d_{2}(t)\right)\right) \\
& +v(t) ;
\end{aligned}
$$

equivalently,

$$
\begin{aligned}
\dot{x}(t) & =-C x(t)+A(x(t)) f(y(t))+\mu_{0} B(x(t)) \\
\cdot & f\left(y\left(t-\tau_{1}(t)\right)\right)+\left(1-\mu_{0}\right) B(x(t)) \\
\cdot & f\left(y\left(t-\tau_{2}(t)\right)\right)+\left(\mu(t)-\mu_{0}\right) B(x(t)) \\
\cdot & \left(f\left(y\left(t-\tau_{1}(t)\right)\right)-f\left(y\left(t-\tau_{2}(t)\right)\right)\right)+u(t), \\
\dot{y}(t) & =-D y(t)+E(y(t)) g(x(t))+\omega_{0} H(y(t)) \\
\cdot & g\left(x\left(t-d_{1}(t)\right)\right)+\left(1-\omega_{0}\right) H(y(t)) \\
\cdot & g\left(x\left(t-d_{2}(t)\right)\right)+\left(\omega(t)-\omega_{0}\right) H(y(t)) \\
\cdot & \left(g\left(x\left(t-d_{1}(t)\right)\right)-g\left(x\left(t-d_{2}(t)\right)\right)\right)+v(t) .
\end{aligned}
$$

Definition 5. System (15) is called passive if there exists a scalar $\gamma>0$ such that

$$
\begin{gathered}
2 \int_{0}^{t_{z}}\left[\begin{array}{lll}
f^{T}(y(s)) & g^{T}(x(s))
\end{array}\right]\left[\begin{array}{l}
u(s) \\
v(s)
\end{array}\right] d s \\
\geq-\gamma \int_{0}^{t_{z}}\left[\begin{array}{ll}
u^{T}(s) & v^{T}(s)
\end{array}\right]\left[\begin{array}{l}
u(s) \\
v(s)
\end{array}\right] d s
\end{gathered}
$$

for all solutions of (15) with $x(0)=0$ and $y(0)=0$ and for all $t_{z} \geq 0$.

Remark 6. Passivity analysis originates from circuit theory and it uses the input-output description method based on energy to design and analyze a system. The physical meaning of passivity is reflected in Definition 5 where the energy growth of the system is always less than or equal to the total energy of the external inputs; this means that the passive system is always accompanied by the loss of energy. In fact, the storage function of the passive system can be used as a Lyapunov function under certain conditions. Thus, both Lyapunov stability theory and passivity theory can be used to research the stability of the system.
Lemma 7. For any scalars $p_{2}>p_{1}>0$, matrix $R \in \Re^{n \times n}, R=$ $R^{T}>0$, and vector function $\omega:\left[p_{1}, p_{2}\right] \mapsto R^{n}$, the inequalities hold as follows:

$$
\begin{aligned}
& \left(p_{2}-p_{1}\right) \int_{p_{1}}^{p_{2}} \omega^{T}(s) R \omega(s) d s \\
& \geq\left(\int_{p_{1}}^{p_{2}} \omega(s) d s\right)^{T} R\left(\int_{p_{1}}^{p_{2}} \omega(s) d s\right), \\
& \frac{\left(p_{2}-p_{1}\right)^{2}}{2} \int_{p_{1}}^{p_{2}} \int_{t+\theta}^{t} \omega^{T}(s) R \omega(s) d s d \theta \\
& \geq\left(\int_{p_{1}}^{p_{2}} \int_{t+\theta}^{t} \omega(s) d s\right)^{T} R\left(\int_{p_{1}}^{p_{2}} \int_{t+\theta}^{t} \omega(s) d s\right) .
\end{aligned}
$$

\section{Main Results}

For derivation convenience, we denote

$$
\begin{aligned}
& A=\max \left\{\left|\underline{a}_{j i}\right|,\left|\bar{a}_{j i}\right|\right\}, \\
& B=\max \left\{\left|\underline{b}_{j i}\right|,\left|\bar{b}_{j i}\right|\right\}, \\
& E=\max \left\{\left|\underline{e}_{i j}\right|,\left|\bar{e}_{i j}\right|\right\}, \\
& H=\max \left\{\left|\underline{h}_{i j}\right|,\left|\bar{h}_{i j}\right|\right\} .
\end{aligned}
$$

Theorem 8. Under Assumptions 1-3, system (16) is passive, if there exist any appropriately dimensional matrices $M_{m}(m=$ $1,2, \ldots, 4), W_{w}(w=1,2, \ldots, 6)$, a scalar $\gamma>0$, and symmetric positive definite matrices $R_{i}(i=1,2), Q_{j}(j=$ $3,4, \ldots, 6), O_{o}(o=3,4, \ldots, 6), P_{k}(k=2,3,6,7), Z_{l}(l=$ $1,2, \ldots, 4)$, and $N_{n}(n=1,2)$, such that the following LMIs hold:

$$
\begin{aligned}
& \Omega_{i, j}=\left(\Omega_{i, j}\right)_{14 \times 14}<0, \\
& \Phi_{i, j}=\left(\Phi_{i, j}\right)_{14 \times 14}<0,
\end{aligned}
$$

where

$$
\begin{aligned}
\Omega_{1,1}= & -R_{1} C-C^{T} R_{1}+Q_{3}+Q_{4}-P_{2}-2 Z_{1}-2 Z_{2} \\
& -M_{1} C-C^{T} M_{1}-2 \varsigma^{-} W_{1} \varsigma^{+} \\
\Omega_{1,4}= & P_{2} \\
\Omega_{1,6}= & -M_{1}-M_{2} C \\
\Omega_{1,7}= & R_{1} A+M_{1} A+W_{1}\left(\varsigma^{-}+\varsigma^{+}\right) \\
\Omega_{1,8}= & \mu_{0}\left(R_{1}+M_{1}\right) B \\
\Omega_{1,9}= & \left(1-\mu_{0}\right)\left(M_{1}+R_{1}\right) B \\
\Omega_{1,12}= & \frac{2}{\tau_{1}} Z_{1}
\end{aligned}
$$




$$
\begin{aligned}
& \Omega_{1,13}=\frac{2}{\tau_{2}-\tau_{1}} Z_{2}, \\
& \Omega_{1,14}=R_{1}+M_{1} \text {, } \\
& \Omega_{2,2}=-\left(1-\mu_{1}\right) Q_{3}-2 \varsigma^{-} W_{2} \varsigma^{+}, \\
& \Omega_{2,8}=-\left(1-\mu_{1}\right) Q_{3}+W_{2}\left(\varsigma^{-}+\varsigma^{+}\right) \text {, } \\
& \Omega_{3,3}=-\left(1-\mu_{2}\right) Q_{5}-2 \varsigma^{-} W_{3} \varsigma^{+}, \\
& \Omega_{3,9}=-\left(1-\mu_{2}\right) Q_{5}+W_{3}\left(\varsigma^{-}+\varsigma^{+}\right), \\
& \Omega_{4,4}=Q_{5}+Q_{6}-Q_{4}-P_{2}-P_{3} \text {, } \\
& \Omega_{4,5}=P_{3} \text {, } \\
& \Omega_{4,10}=Q_{5}+Q_{6}-Q_{4} \text {, } \\
& \Omega_{5,5}=-Q_{6}-P_{3} \text {, } \\
& \Omega_{5,11}=-Q_{6} \text {, } \\
& \Omega_{6,6}=\tau_{1}^{2} P_{2}+\left(\tau_{2}-\tau_{1}\right)^{2} P_{3}+\frac{\tau_{1}^{2}}{2} Z_{1}+\frac{\tau_{2}^{2}-\tau_{1}^{2}}{2} Z_{2} \\
& -2 M_{2}, \\
& \Omega_{6,7}=M_{2} A \text {, } \\
& \Omega_{6,8}=\mu_{0} M_{2} B, \\
& \Omega_{6,9}=\left(1-\mu_{0}\right) M_{2} B, \\
& \Omega_{6,14}=M_{2} \text {, } \\
& \Omega_{7,7}=-2 W_{1}, \\
& \Omega_{7,14}=-I \text {, } \\
& \Omega_{8,8}=-\left(1-\mu_{1}\right) Q_{3}-2 W_{2}, \\
& \Omega_{9,9}=-\left(1-\mu_{2}\right) Q_{5}-2 W_{3}, \\
& \Omega_{10,10}=Q_{5}+Q_{6}-Q_{4} \text {, } \\
& \Omega_{11,11}=-Q_{6} \text {, } \\
& \Omega_{12,12}=-\frac{2}{\tau_{1}^{2}} Z_{1}, \\
& \Omega_{13,13}=-\frac{2}{\left(\tau_{2}-\tau_{1}\right)^{2}} Z_{2}, \\
& \Omega_{14,14}=-\gamma I \text {, } \\
& \Phi_{1,1}=-R_{2} D-D^{T} R_{2}+O_{3}+O_{4}-P_{6}-2 Z_{3}-2 Z_{4} \\
& -M_{3} D-D^{T} M_{3}-2 \sigma^{-} W_{4} \sigma^{+} \text {, } \\
& \Phi_{1,4}=P_{6}, \\
& \Phi_{1,6}=-M_{3}-M_{4} D \text {, } \\
& \Phi_{1,7}=R_{2} E+M_{3} E+W_{4}\left(\sigma^{-}+\sigma^{+}\right),
\end{aligned}
$$

$$
\begin{aligned}
& \Phi_{1,8}=\omega_{0}\left(R_{2}+M_{3}\right) H, \\
& \Phi_{1,9}=\left(1-\omega_{0}\right)\left(M_{3}+R_{2}\right) H, \\
& \Phi_{1,12}=\frac{2}{d_{1}} Z_{3}, \\
& \Phi_{1,13}=\frac{2}{d_{2}-d_{1}} Z_{4}, \\
& \Phi_{1,14}=R_{2}+M_{3} \\
& \Phi_{2,2}=-\left(1-\omega_{1}\right) O_{3}-2 \sigma^{-} W_{5} \sigma^{+}, \\
& \Phi_{2,8}=-\left(1-\omega_{1}\right) O_{3}+W_{5}\left(\sigma^{-}+\sigma^{+}\right) \text {, } \\
& \Phi_{3,3}=-\left(1-\omega_{2}\right) O_{5}-2 \sigma^{-} W_{6} \sigma^{+} \text {, } \\
& \Phi_{3,9}=-\left(1-\omega_{2}\right) O_{5}+W_{6}\left(\sigma^{-}+\sigma^{+}\right) \text {, } \\
& \Phi_{4,4}=O_{5}+O_{6}-O_{4}-P_{6}-P_{7} \text {, } \\
& \Phi_{4,5}=P_{7}, \\
& \Phi_{4,10}=\mathrm{O}_{5}+\mathrm{O}_{6}-\mathrm{O}_{4} \text {, } \\
& \Phi_{5,5}=-\mathrm{O}_{6}-P_{7}, \\
& \Phi_{5,11}=-\mathrm{O}_{6} \text {, } \\
& \Phi_{6,6}=d_{1}^{2} P_{6}+\left(d_{2}-d_{1}\right)^{2} P_{7}+\frac{d_{1}^{2}}{2} Z_{3}+\frac{d_{2}^{2}-d_{1}^{2}}{2} Z_{4} \\
& -2 M_{4} \\
& \Phi_{6,7}=M_{4} E, \\
& \Phi_{6,8}=\omega_{0} M_{4} H, \\
& \Phi_{6,9}=\left(1-\omega_{0}\right) M_{4} H, \\
& \Phi_{6,14}=M_{4} \text {, } \\
& \Phi_{7,7}=-2 W_{4}, \\
& \Phi_{7,14}=-I \text {, } \\
& \Phi_{8,8}=-\left(1-\omega_{1}\right) O_{3}-2 W_{5}, \\
& \Phi_{9,9}=-\left(1-\omega_{2}\right) O_{5}-2 W_{6} \text {, } \\
& \Phi_{10,10}=\mathrm{O}_{5}+\mathrm{O}_{6}-\mathrm{O}_{4} \text {, } \\
& \Phi_{11,11}=-\mathrm{O}_{6} \text {, } \\
& \Phi_{12,12}=-\frac{2}{d_{1}^{2}} Z_{3}, \\
& \Phi_{13,13}=-\frac{2}{\left(d_{2}-d_{1}\right)^{2}} Z_{4} \text {, } \\
& \Phi_{14,14}=-\gamma I .
\end{aligned}
$$


Proof. Consider the following LKF candidate:

$$
V(t)=\sum_{i=1}^{8} V_{i}(t)
$$

where

$$
\begin{aligned}
& V_{1}(t)=x^{T}(t) R_{1} x(t), \\
& V_{2}(t)=y^{T}(t) R_{2} y(t) \text {, } \\
& V_{3}(t)=\int_{t-\tau_{1}(t)}^{t}\left[\begin{array}{c}
x(s) \\
f(y(s))
\end{array}\right]^{T} Q_{3}\left[\begin{array}{c}
x(s) \\
f(y(s))
\end{array}\right] d s \\
& +\int_{t-\tau_{1}}^{t}\left[\begin{array}{c}
x(s) \\
f(y(s))
\end{array}\right]^{T} Q_{4}\left[\begin{array}{c}
x(s) \\
f(y(s))
\end{array}\right] d s \\
& +\int_{t-\tau_{2}(t)}^{t-\tau_{1}}\left[\begin{array}{c}
x(s) \\
f(y(s))
\end{array}\right]^{T} Q_{5}\left[\begin{array}{c}
x(s) \\
f(y(s))
\end{array}\right] d s \\
& +\int_{t-\tau_{2}}^{t-\tau_{1}}\left[\begin{array}{c}
x(s) \\
f(y(s))
\end{array}\right]^{T} Q_{6}\left[\begin{array}{c}
x(s) \\
f(y(s))
\end{array}\right] d s, \\
& V_{4}(t)=\int_{t-d_{1}(t)}^{t}\left[\begin{array}{c}
y(s) \\
g(x(s))
\end{array}\right]^{T} O_{3}\left[\begin{array}{c}
y(s) \\
g(x(s))
\end{array}\right] d s \\
& +\int_{t-d_{1}}^{t}\left[\begin{array}{c}
y(s) \\
g(x(s))
\end{array}\right]^{T} O_{4}\left[\begin{array}{c}
y(s) \\
g(x(s))
\end{array}\right] d s \\
& +\int_{t-d_{2}(t)}^{t-d_{1}}\left[\begin{array}{c}
y(s) \\
g(x(s))
\end{array}\right]^{T} O_{5}\left[\begin{array}{c}
y(s) \\
g(x(s))
\end{array}\right] d s \\
& +\int_{t-d_{2}}^{t-d_{1}}\left[\begin{array}{c}
y(s) \\
g(x(s))
\end{array}\right]^{T} O_{6}\left[\begin{array}{c}
y(s) \\
g(x(s))
\end{array}\right] d s, \\
& V_{5}(t)=\tau_{1} \int_{-\tau_{1}}^{0} \int_{t+\theta}^{t} \dot{x}^{T}(s) P_{2} \dot{x}(s) d s d \theta \\
& +\left(\tau_{2}-\tau_{1}\right) \int_{-\tau_{2}}^{-\tau_{1}} \int_{t+\theta}^{t} \dot{x}^{T}(s) P_{3} \dot{x}(s) d s d \theta, \\
& V_{6}(t)=d_{1} \int_{-d_{1}}^{0} \int_{t+\theta}^{t} \dot{y}^{T}(s) P_{6} \dot{y}(s) d s d \theta \\
& +\left(d_{2}-d_{1}\right) \int_{-d_{2}}^{-d_{1}} \int_{t+\theta}^{t} \dot{y}^{T}(s) P_{7} \dot{y}(s) d s d \theta, \\
& V_{7}(t)=\int_{-\tau_{1}}^{0} \int_{\theta}^{0} \int_{t+\lambda}^{t} \dot{x}^{T}(s) Z_{1} \dot{x}(s) d s d \lambda d \theta \\
& +\int_{-\tau_{2}}^{-\tau_{1}} \int_{\theta}^{0} \int_{t+\lambda}^{t} \dot{x}^{T}(s) Z_{2} \dot{x}(s) d s d \lambda d \theta, \\
& V_{8}(t)=\int_{-d_{1}}^{0} \int_{\theta}^{0} \int_{t+\lambda}^{t} \dot{y}^{T}(s) Z_{3} \dot{y}(s) d s d \lambda d \theta \\
& +\int_{-d_{2}}^{-d_{1}} \int_{\theta}^{0} \int_{t+\lambda}^{t} \dot{y}^{T}(s) Z_{4} \dot{y}(s) d s d \lambda d \theta .
\end{aligned}
$$

Then, we define the infinitesimal generator $\mathscr{L}$ of $V(t)$ as

$$
\mathscr{L} V(t)=\lim _{\Delta \rightarrow 0^{+}} \frac{1}{\Delta}\left\{\mathbb{E}\left(\frac{V(t+\Delta)}{t}\right)-V(t)\right\} .
$$

Taking the mathematical expectation of $V(t)$, we get

$$
\begin{aligned}
& \mathbb{E}\{\left.\mathscr{L} V_{1}(t)\right\}=\mathbb{E}\left\{2 x^{T}(t) R_{1}(-C x(t)+A(x(t))\right. \\
& \cdot f(y(t))+\mu_{0} B(x(t)) f\left(y\left(t-\tau_{1}(t)\right)\right) \\
&+\left(1-\mu_{0}\right) B(x(t)) f\left(y\left(t-\tau_{2}(t)\right)\right)+\left(\mu(t)-\mu_{0}\right) \\
& \cdot B(x(t))\left(f\left(y\left(t-\tau_{1}(t)\right)\right)-f\left(y\left(t-\tau_{2}(t)\right)\right)\right) \\
&+u(t))\} \leq \mathbb{E}\left\{2 x^{T}(t) R_{1}(-C x(t)+A f(y(t))\right. \\
&+ \mu_{0} B f\left(y\left(t-\tau_{1}(t)\right)\right)+\left(1-\mu_{0}\right) \\
& \cdot\left.\left.B f\left(y\left(t-\tau_{2}(t)\right)\right)+u(t)\right)\right\}, \\
& \mathbb{E}\left\{\mathscr{L} V_{2}(t)\right\}=\mathbb{E}\left\{2 y^{T}(t) R_{2}(-D y(t)+E(y(t))\right. \\
& \cdot g(x(t))+\omega_{0} H(y(t)) g\left(x\left(t-d_{1}(t)\right)\right) \\
&\left.+\left(1-\omega_{0}\right) H(y(t)) g\left(x\left(t-d_{2}(t)\right)\right)\right)+(\omega(t) \\
&\left.-\omega_{0}\right) H(y(t))\left(g\left(x\left(t-d_{1}(t)\right)\right)\right. \\
&\left.\left.-g\left(x\left(t-d_{2}(t)\right)\right)\right)+v(t)\right\} \leq \mathbb{E}\left\{2 y^{T}(t)\right. \\
&+R_{2}\left(-D y(t)+E g(x(t))+\omega_{0} H g\left(x\left(t-d_{1}(t)\right)\right)\right. \\
&\left.\left.+\left(1-\omega_{0}\right) H g\left(x\left(t-d_{2}(t)\right)\right)+v(t)\right)\right\} .
\end{aligned}
$$

According to Assumption 3, we obtain

$$
\begin{gathered}
\mathbb{E}\left\{\mathscr{L} V_{3}(t)\right\} \leq \mathbb{E}\left\{x^{T}(t)\left(Q_{3}+Q_{4}\right) x(t)-\left(1-\mu_{1}\right)\right. \\
\cdot\left[\begin{array}{c}
x\left(t-\tau_{1}(t)\right) \\
f\left(y\left(t-\tau_{1}(t)\right)\right)
\end{array}\right]^{T} Q_{3}\left[\begin{array}{c}
x\left(t-\tau_{1}(t)\right) \\
f\left(y\left(t-\tau_{1}(t)\right)\right)
\end{array}\right] \\
+\left[\begin{array}{c}
x\left(t-\tau_{1}\right) \\
f\left(y\left(t-\tau_{1}\right)\right)
\end{array}\right]^{T}\left(Q_{5}+Q_{6}-Q_{4}\right) \\
\cdot\left[\begin{array}{c}
x\left(t-\tau_{1}\right) \\
f\left(y\left(t-\tau_{1}\right)\right)
\end{array}\right]-\left(1-\mu_{2}\right)\left[\begin{array}{c}
x\left(t-\tau_{2}(t)\right) \\
f\left(y\left(t-\tau_{2}(t)\right)\right)
\end{array}\right]^{T} \\
\cdot Q_{5}\left[\begin{array}{c}
x\left(t-\tau_{2}(t)\right) \\
f\left(y\left(t-\tau_{2}(t)\right)\right)
\end{array}\right]-\left[\begin{array}{c}
x\left(t-\tau_{2}\right) \\
f\left(y\left(t-\tau_{2}\right)\right)
\end{array}\right]^{T} \\
\left.\cdot Q_{6}\left[\begin{array}{c}
x\left(t-\tau_{2}\right) \\
f\left(y\left(t-\tau_{2}\right)\right)
\end{array}\right]\right\}
\end{gathered}
$$


8

Mathematical Problems in Engineering

$$
\begin{aligned}
& \mathbb{E}\left\{\mathscr{L} V_{4}(t)\right\} \leq \mathbb{E}\left\{y^{T}(t)\left(O_{3}+O_{4}\right) y(t)-\left(1-\omega_{1}\right)\right. \\
& \cdot\left[\begin{array}{c}
y\left(t-d_{1}(t)\right) \\
g\left(x\left(t-d_{1}(t)\right)\right)
\end{array}\right]^{T} O_{3}\left[\begin{array}{c}
y\left(t-d_{1}(t)\right) \\
g\left(x\left(t-d_{1}(t)\right)\right)
\end{array}\right] \\
& +\left[\begin{array}{c}
y\left(t-d_{1}\right) \\
g\left(x\left(t-d_{1}\right)\right)
\end{array}\right]^{T}\left(O_{5}+O_{6}-O_{4}\right) \\
& \cdot\left[\begin{array}{c}
y\left(t-d_{1}\right) \\
g\left(x\left(t-d_{1}\right)\right)
\end{array}\right]-\left(1-\omega_{2}\right)\left[\begin{array}{c}
y\left(t-d_{2}(t)\right) \\
g\left(x\left(t-d_{2}(t)\right)\right)
\end{array}\right]^{T} \\
& \cdot O_{5}\left[\begin{array}{c}
y\left(t-d_{2}(t)\right) \\
g\left(x\left(t-d_{2}(t)\right)\right)
\end{array}\right]-\left[\begin{array}{c}
y\left(t-\tau_{2}\right) \\
g\left(x\left(t-\tau_{2}\right)\right)
\end{array}\right]^{T} \\
& \left.\cdot O_{6}\left[\begin{array}{c}
y\left(t-\tau_{2}\right) \\
g\left(x\left(t-\tau_{2}\right)\right)
\end{array}\right]\right\} .
\end{aligned}
$$

It is obvious that

$$
\begin{aligned}
& \mathbb{E}\left\{\mathscr{L} V_{5}(t)\right\}=\mathbb{E}\left\{\dot{x}^{T}(t)\left[\tau_{1}^{2} P_{2}+\left(\tau_{2}-\tau_{1}\right)^{2} P_{3}\right] \dot{x}(t)\right. \\
& -\tau_{1} \int_{t-\tau_{1}}^{t} \dot{x}^{T}(s) P_{2} \dot{x}(s) d s \\
& \left.-\left(\tau_{2}-\tau_{1}\right) \int_{t-\tau_{2}}^{t-\tau_{1}} \dot{x}^{T}(s) P_{3} \dot{x}(s) d s\right\}, \\
& \mathbb{E}\left\{\mathscr{L} V_{6}(t)\right\}=\mathbb{E}\left\{\dot{y}^{T}(t)\left[d_{1}^{2} P_{6}+\left(d_{2}-d_{1}\right)^{2} P_{7}\right] \dot{y}(t)\right. \\
& -d_{1} \int_{t-d_{1}}^{t} \dot{y}^{T}(s) P_{6} \dot{y}(s) d s \\
& \left.-\left(d_{2}-d_{1}\right) \int_{t-d_{2}}^{t-d_{1}} \dot{y}^{T}(s) P_{7} \dot{y}(s) d s\right\} .
\end{aligned}
$$

Using Lemma 7, we have

$$
\begin{aligned}
& -\tau_{1} \int_{t-\tau_{1}}^{t} \dot{x}^{T}(s) P_{2} \dot{x}(s) d s \leq-\left(\int_{t-\tau_{1}}^{t} \dot{x}(s) d s\right)^{T} \\
& \cdot P_{2}\left(\int_{t-\tau_{1}}^{t} \dot{x}(s) d s\right)=-\left[x(t)-x\left(t-\tau_{1}\right)\right]^{T} \\
& \cdot P_{2}\left[x(t)-x\left(t-\tau_{1}\right)\right] \\
& -\left(\tau_{2}-\tau_{1}\right) \int_{t-\tau_{2}}^{t-\tau_{1}} \dot{x}^{T}(s) P_{3} \dot{x}(s) d s \\
& \leq-\left(\int_{t-\tau_{2}}^{t-\tau_{1}} \dot{x}(s) d s\right)^{T} P_{3}\left(\int_{t-\tau_{2}}^{t-\tau_{1}} \dot{x}(s) d s\right)
\end{aligned}
$$

$$
\begin{aligned}
& =-\left[x\left(t-\tau_{1}\right)-x\left(t-\tau_{2}\right)\right]^{T} \\
& \cdot P_{3}\left[x\left(t-\tau_{1}\right)-x\left(t-\tau_{2}\right)\right] .
\end{aligned}
$$

Similarly,

$$
\begin{aligned}
& -d_{1} \int_{t-d_{1}}^{t} \dot{y}^{T}(s) P_{6} \dot{y}(s) d s \leq-\left(\int_{t-d_{1}}^{t} \dot{y}(s) d s\right)^{T} \\
& \cdot P_{6}\left(\int_{t-d_{1}}^{t} \dot{y}(s) d s\right)=-\left[y(t)-y\left(t-d_{1}\right)\right]^{T} \\
& \cdot P_{6}\left[y(t)-y\left(t-d_{1}\right)\right], \\
& -\left(d_{2}-d_{1}\right) \int_{t-d_{2}}^{t-d_{1}} \dot{y}^{T}(s) P_{7} \dot{y}(s) d s \\
& \leq-\left(\int_{t-d_{2}}^{t-d_{1}} \dot{y}(s) d s\right)^{T} P_{7}\left(\int_{t-d_{2}}^{t-d_{1}} \dot{y}(s) d s\right) \\
& =-\left[y\left(t-d_{1}\right)-y\left(t-d_{2}\right)\right]^{T} \\
& \cdot P_{7}\left[y\left(t-d_{1}\right)-y\left(t-d_{2}\right)\right] .
\end{aligned}
$$

Moreover, we obtain

$$
\begin{aligned}
& \mathbb{E}\left\{\mathscr{L} V_{7}(t)\right\}=\mathbb{E}\left\{\dot{x}^{T}(t)\left(\frac{\tau_{1}^{2}}{2} Z_{1}+\frac{\tau_{2}^{2}-\tau_{1}^{2}}{2} Z_{2}\right) \dot{x}(t)\right. \\
& -\int_{-\tau_{1}}^{0} \int_{t+\theta}^{t} \dot{x}^{T}(s) Z_{1} \dot{x}(s) d s d \theta \\
& \left.-\int_{-\tau_{2}}^{-\tau_{1}} \int_{t+\theta}^{t} \dot{x}^{T}(s) Z_{2} \dot{x}(s) d s d \theta\right\}, \\
& \mathbb{E}\left\{\mathscr{L} V_{8}(t)\right\}=\mathbb{E}\left\{\dot{y}^{T}(t)\left(\frac{d_{1}^{2}}{2} Z_{3}+\frac{d_{2}^{2}-d_{1}^{2}}{2} Z_{4}\right) \dot{y}(t)\right. \\
& -\int_{-d_{1}}^{0} \int_{t+\theta}^{t} \dot{y}^{T}(s) Z_{3} \dot{y}(s) d s d \theta \\
& \left.-\int_{-d_{2}}^{-d_{1}} \int_{t+\theta}^{t} \dot{y}^{T}(s) Z_{4} \dot{y}(s) d s d \theta\right\} .
\end{aligned}
$$

Using (18), we have

$$
\begin{aligned}
& -\int_{-\tau_{1}}^{0} \int_{t+\theta}^{t} \dot{x}^{T}(s) Z_{1} \dot{x}(s) d s d \theta \\
& \leq-\frac{2}{\tau_{1}^{2}}\left(\int_{-\tau_{1}}^{0} \int_{t+\theta}^{t} \dot{x}(s) d s d \theta\right)^{T} \\
& \cdot Z_{1}\left(\int_{-\tau_{1}}^{0} \int_{t+\theta}^{t} \dot{x}(s) d s d \theta\right)=-2 x^{T}(t) Z_{1} x(t) \\
& +\frac{4}{\tau_{1}} x^{T}(t) Z_{1}\left(\int_{t-\tau_{1}}^{t} x(s) d s\right) \\
& -\frac{2}{\tau_{1}^{2}}\left(\int_{t-\tau_{1}}^{t} x(s) d s\right)^{T} Z_{1}\left(\int_{t-\tau_{1}}^{t} x(s) d s\right),
\end{aligned}
$$




$$
\begin{aligned}
& -\int_{-\tau_{2}}^{-\tau_{1}} \int_{t+\theta}^{t} \dot{x}^{T}(s) Z_{2} \dot{x}(s) d s d \theta \\
& \leq-\frac{2}{\left(\tau_{2}-\tau_{1}\right)^{2}}\left(\int_{-\tau_{2}}^{-\tau_{1}} \int_{t+\theta}^{t} \dot{x}(s) d s d \theta\right)^{T} \\
& \quad \times Z_{2}\left(\int_{-\tau_{2}}^{-\tau_{1}} \int_{t+\theta}^{t} \dot{x}(s) d s d \theta\right)=-2 x^{T}(t) Z_{2} x(t) \\
& +\frac{4}{\tau_{2}-\tau_{1}} x^{T}(t) Z_{2}\left(\int_{t-\tau_{2}}^{t-\tau_{1}} x(s) d s\right) \\
& -\frac{2}{\left(\tau_{2}-\tau_{1}\right)^{2}}\left(\int_{t-\tau_{2}}^{t-\tau_{1}} x(s) d s\right)^{T} Z_{2}\left(\int_{t-\tau_{2}}^{t-\tau_{1}} x(s) d s\right) .
\end{aligned}
$$

Similarly,

$$
\begin{aligned}
& -\int_{-d_{1}}^{0} \int_{t+\theta}^{t} \dot{y}^{T}(s) Z_{3} \dot{y}(s) d s d \theta \\
& \leq-\frac{2}{d_{1}^{2}}\left(\int_{-d_{1}}^{0} \int_{t+\theta}^{t} \dot{y}(s) d s d \theta\right)^{T} \\
& \cdot Z_{3}\left(\int_{-d_{1}}^{0} \int_{t+\theta}^{t} \dot{y}(s) d s d \theta\right)=-2 y^{T}(t) Z_{3} y(t) \\
& +\frac{4}{d_{1}} y^{T}(t) Z_{3}\left(\int_{t-d_{1}}^{t} y(s) d s\right) \\
& -\frac{2}{d_{1}^{2}}\left(\int_{t-d_{1}}^{t} y(s) d s\right)^{T} Z_{3}\left(\int_{t-d_{1}}^{t} y(s) d s\right) \\
& -\int_{-d_{2}}^{-d_{1}} \int_{t+\theta}^{t} y^{T}(s) Z_{4} y(s) d s d \theta \\
& \leq-\frac{2}{\left(d_{2}-d_{1}\right)^{2}}\left(\int_{-d_{2}}^{-d_{1}} \int_{t+\theta}^{t} y(s) d s d \theta\right)^{T} \\
& \times Z_{4}\left(\int_{-d_{2}}^{-d_{1}} \int_{t+\theta}^{t} y(s) d s d \theta\right)=-2 y^{T}(t) Z_{4} y(t) \\
& +\frac{4}{d_{2}-d_{1}} y^{T}(t) Z_{4}\left(\int_{t-d_{2}}^{t-d_{1}} y(s) d s\right) \\
& -\frac{2}{\left(d_{2}-d_{1}\right)^{2}}\left(\int_{t-d_{2}}^{t-d_{1}} y(s) d s\right)^{T}\left(\int_{t-d_{2}}^{t-d_{1}} y(s) d s\right) .
\end{aligned}
$$

To derive a less conservative criterion, we add the following inequations with any matrices $M_{1}, M_{2}, M_{3}$, and $M_{4}$ of appropriate dimensions:

$$
\begin{aligned}
0 & \leq 2\left(x^{T}(t) M_{1}+\dot{x}^{T}(t) M_{2}\right)[-\dot{x}-C x(t) \\
& +A f(y(t))+\mu_{0} B f\left(y\left(t-\tau_{1}(t)\right)\right)
\end{aligned}
$$

$$
\begin{aligned}
& \left.+\left(1-\mu_{0}\right) B f\left(y\left(t-\tau_{2}(t)\right)\right)+u(t)\right], \\
0 \leq & 2\left(y^{T}(t) M_{3}+\dot{y}^{T}(t) M_{4}\right)[-\dot{y}-D y(t) \\
& +E g(x(t))+\omega_{0} H g\left(x\left(t-d_{1}(t)\right)\right) \\
& \left.+\left(1-\omega_{0}\right) H g\left(x\left(t-d_{2}(t)\right)\right)+v(t)\right] .
\end{aligned}
$$

Based on Assumption 1 [20], we have

$$
\begin{aligned}
& \varsigma^{-} x(t)-f(y(t)) \leq 0, \\
& f(y(t))-\varsigma^{+} x(t) \leq 0, \\
& \sigma^{-} y(t)-g(x(t)) \leq 0, \\
& g(x(t))-\sigma^{+} y(t) \leq 0,
\end{aligned}
$$

and there exist positive diagonal matrices $W_{i}>0(i=$ $1,2, \ldots, 8)$; the following inequalities hold:

$$
\begin{aligned}
0 \leq 2 & {\left[\varsigma^{-} x(t)-f(y(t))\right]^{T} W_{1}\left[f(y(t))-\varsigma^{+} x(t)\right] } \\
\leq & -2 f^{T}(y(t)) W_{1} f(y(t)) \\
& +2 x^{T}(t) W_{1}\left(\varsigma^{-}+\varsigma^{+}\right) f(y(t)) \\
& -2 x^{T}(t) \varsigma^{-} W_{1} \varsigma^{+} x(t) .
\end{aligned}
$$

Similarly,

$$
\begin{aligned}
0 \leq & -2 f^{T}\left(y\left(t-\tau_{1}\right)\right) W_{2} f\left(y\left(t-\tau_{1}\right)\right) \\
& +2 x(t)^{T}\left(t-\tau_{1}\right) W_{2}\left(\varsigma^{-}+\varsigma^{+}\right) f\left(y\left(t-\tau_{1}\right)\right) \\
& -2 x^{T}\left(t-\tau_{1}\right) \varsigma^{-} W_{2} \varsigma^{+} x\left(t-\tau_{1}\right), \\
0 \leq & -2 f^{T}\left(y\left(t-\tau_{2}\right)\right) W_{3} f\left(y\left(t-\tau_{2}\right)\right) \\
& +2 x^{T}\left(t-\tau_{2}\right) W_{3}\left(\varsigma^{-}+\varsigma^{+}\right) f\left(y\left(t-\tau_{2}\right)\right) \\
& -2 x^{T}\left(t-\tau_{2}\right) \varsigma^{-} W_{3} \varsigma^{+} x\left(t-\tau_{2}\right), \\
0 \leq & 2\left[\sigma^{-} y(t)-g(x(t))\right]^{T} W_{4}\left[g(x(t))-\sigma^{+} y(t)\right] \\
\leq & -2 g^{T}(x(t)) W_{4} g(x(t)) \\
& +2 y^{T}(t) W_{4}\left(\sigma^{-}+\sigma^{+}\right) g(x(t)) \\
& -2 y^{T}(t) \sigma^{-} W_{4} \sigma^{+} y(t), \\
0 \leq & -2 g^{T}\left(x\left(t-d_{1}\right)\right) W_{5} g\left(x\left(t-d_{1}\right)\right) \\
& +2 y(t)^{T}\left(t-d_{1}\right) W_{5}\left(\sigma^{-}+\sigma^{+}\right) g\left(x\left(t-d_{1}\right)\right) \\
& -2 y^{T}\left(t-d_{1}\right) \sigma^{-} W_{5} \sigma^{+} y\left(t-d_{1}\right), \\
0 \leq & -2 g^{T}\left(x\left(t-d_{2}\right)\right) W_{6} g\left(x\left(t-d_{2}\right)\right) \\
& +2 y^{T}\left(t-d_{2}\right) W_{6}\left(\sigma^{-}+\sigma^{+}\right) g\left(x\left(t-d_{2}\right)\right) \\
& -2 y^{T}\left(t-d_{2}\right) \sigma^{-} W_{6} \sigma^{+} y\left(t-d_{2}\right) .
\end{aligned}
$$


Define

$$
\begin{aligned}
& \xi(t)=\left\{\begin{array}{lll}
x^{T}(t) & x^{T}\left(t-\tau_{1}(t)\right) & x^{T}\left(t-\tau_{2}(t)\right)
\end{array}\right. \\
& x^{T}\left(t-\tau_{1}\right) \quad x^{T}\left(t-\tau_{2}\right) \quad \dot{x}(t) \quad f^{T}(y(t)) \\
& f^{T}\left(y\left(t-\tau_{1}(t)\right)\right) f^{T}\left(y\left(t-\tau_{2}(t)\right)\right) f^{T}\left(y\left(t-\tau_{1}\right)\right) \\
& \left.f^{T}\left(y\left(t-\tau_{2}\right)\right) \int_{t-\tau_{1}}^{t} x^{T}(s) d s \int_{t-\tau_{2}}^{t-\tau_{1}} x^{T}(s) d s \quad u^{T}(t)\right\}, \\
& \zeta(t)=\left\{\begin{array}{lll}
y^{T}(t) & y^{T}\left(t-d_{1}(t)\right) & y^{T}\left(t-d_{2}(t)\right)
\end{array}\right. \\
& \begin{array}{lllll}
y^{T}\left(t-d_{1}\right) & y^{T}\left(t-d_{2}\right) & \dot{y}(t) & g^{T}(x(t)) & g^{T}\left(x\left(t-d_{1}(t)\right)\right)
\end{array} \\
& g^{T}\left(x\left(t-d_{2}(t)\right)\right) \quad g^{T}\left(x\left(t-d_{1}\right)\right) \quad g^{T}\left(x\left(t-d_{2}\right)\right) \\
& \left.\int_{t-d_{1}}^{t} y^{T}(s) d s \int_{t-d_{2}}^{t-d_{1}} y^{T}(s) d s v^{T}(t)\right\} .
\end{aligned}
$$

Combining (23), (27)-(37), and (40), we have

$$
\begin{aligned}
& \mathbb{E}\left\{\mathscr{L} V(t)-2\left[f^{T}(y(s)) g^{T}(x(s))\right]\left[\begin{array}{l}
u(s) \\
v(s)
\end{array}\right]\right. \\
& \left.\quad-\gamma\left[u^{T}(t) v^{T}(t)\right]\left[\begin{array}{l}
u(t) \\
v(t)
\end{array}\right] d s\right\} \leq \mathbb{E}\left\{\xi^{T}(t) \Omega \xi(t)\right. \\
& \left.+\zeta^{T}(t) \Phi \zeta(t)\right\} .
\end{aligned}
$$

Thus, we conclude that if (42) holds, then

$$
\begin{gathered}
\mathbb{E}\left\{\mathscr{L} V(t)-2\left[\begin{array}{ll}
f^{T}(y(s)) & g^{T}(x(s))
\end{array}\right]\left[\begin{array}{l}
u(s) \\
v(s)
\end{array}\right]\right. \\
\left.-\gamma\left[\begin{array}{ll}
u^{T}(t) & v^{T}(t)
\end{array}\right]\left[\begin{array}{l}
u(t) \\
v(t)
\end{array}\right] d s\right\} \leq 0 .
\end{gathered}
$$

By integrating (43) about $t$ from 0 to $t_{z}$, we get

$$
\begin{aligned}
2 \mathbb{E} \int_{0}^{t_{z}} & {\left[f^{T}(y(s)) g^{T}(x(s))\right]\left[\begin{array}{l}
u(s) \\
v(s)
\end{array}\right] d s } \\
\geq & \mathbb{E} V(t)-\mathbb{E} V(0) \\
& -\gamma \mathbb{E} \int_{0}^{t_{z}}\left[\begin{array}{ll}
u^{T}(s) & v^{T}(s)
\end{array}\right]\left[\begin{array}{l}
u(s) \\
v(s)
\end{array}\right] d s \\
\geq & -\gamma \mathbb{E} \int_{0}^{t_{z}}\left[\begin{array}{ll}
u^{T}(s) & v^{T}(s)
\end{array}\right]\left[\begin{array}{l}
u(s) \\
v(s)
\end{array}\right] d s .
\end{aligned}
$$

Therefore, from Definition 5, we know that the MBAMNN is passive. This completes the proof.

Considering the MBAMNN with leakage, probabilistic, and distributed time-varying delays, then model (1) becomes

$$
\begin{aligned}
\dot{x}_{i}(t)= & -C_{i} x_{i}(t-\delta(t))+\sum_{j=1}^{m} a_{j i}\left(x_{i}(t)\right) f_{j}\left(y_{j}(t)\right) \\
& +\sum_{j=1}^{m} b_{j i}\left(x_{i}(t)\right) f_{j}\left(y_{j}(t-\tau(t))\right)
\end{aligned}
$$

$$
\begin{aligned}
& +\sum_{j=1}^{m} k_{j i}\left(x_{i}(t)\right) \int_{t-\alpha(t)}^{t} f_{j}\left(y_{j}(s)\right) d s \\
& +u_{i}(t), \\
\dot{y}_{j}(t)= & -D_{j} y_{j}(t-\rho(t))+\sum_{i=1}^{n} e_{i j}\left(y_{j}(t)\right) g_{i}\left(x_{i}(t)\right) \\
& +\sum_{i=1}^{n} h_{i j}\left(y_{j}(t)\right) g_{i}\left(x_{i}(t-d(t))\right) \\
& +\sum_{i=1}^{n} l_{i j}\left(y_{j}(t)\right) \int_{t-\beta(t)}^{t} g_{i}\left(x_{i}(s)\right) d s \\
& +v_{j}(t),
\end{aligned}
$$

where the nonnegative continuous variables $\delta(t), \rho(t)$ and $\alpha(t), \beta(t)$ correspond to the leakage and distributed timevarying delays and $K(x(t))=\left(k_{j i}\left(x_{i}(t)\right)\right)_{m \times n}$ and $L(x(t))=$ $\left(l_{i j}\left(y_{j}(t)\right)\right)_{n \times m}$ are the delayed connection weight matrix functions. Then, we let

$$
\begin{aligned}
& k_{j i}\left(x_{i}(t)\right)= \begin{cases}\widehat{b}_{j i}, & \left|x_{i}(t)\right|>\Theta_{i}, \\
\check{b}_{j i}, & \left|x_{i}(t)\right| \leq \Theta_{i},\end{cases} \\
& l_{i j}\left(y_{j}(t)\right)= \begin{cases}\widehat{f}_{i j}, & \left|y_{j}(t)\right|>\Psi_{j}, \\
\check{f}_{i j}, & \left|y_{j}(t)\right| \leq \Psi_{j},\end{cases}
\end{aligned}
$$

in which $k_{j i}$ and $l_{i j}$ are known constants and $k_{j i}\left(x_{i}(t)\right)$ and $l_{i j}\left(y_{j}(t)\right)$ are piecewise continuous functions; we set

$$
\begin{aligned}
\bar{k}_{j i} & =\max \left\{\widehat{k}_{j i}, \check{k}_{j i}\right\}, \\
\underline{k}_{j i} & =\min \left\{\widehat{k}_{j i}, \check{k}_{j i}\right\}, \\
\bar{l}_{i j} & =\max \left\{\hat{l}_{i j}, \check{l}_{i j}\right\}, \\
\underline{l}_{i j} & =\min \left\{\widehat{l}_{i j}, \check{l}_{i j}\right\} .
\end{aligned}
$$

Then, the set-valued maps are defined as

$$
\begin{array}{r}
\operatorname{co}\left[k_{j i}\left(x_{i}(t)\right)\right]= \begin{cases}\widehat{k}_{j i}, & \left|x_{i}(t)\right|>\Theta_{i}, \\
{\left[\underline{k}_{j i}, \bar{k}_{j i}\right],} & \left|x_{i}(t)\right|=\Theta_{i}, \\
\check{k}_{j i}, & \left|x_{i}(t)\right|<\Theta_{i},\end{cases} \\
\operatorname{co}\left[l_{i j}\left(y_{j}(t)\right)\right]= \begin{cases}\hat{l}_{i j}, & \left|y_{j}(t)\right|>\Psi_{j}, \\
{\left[l_{i j}, \bar{l}_{i j}\right],} & \left|y_{j}(t)\right|=\Psi_{j}, \\
\check{l}_{i j}, & \left|y_{j}(t)\right|<\Psi_{j} .\end{cases}
\end{array}
$$


By applying the theories of set-valued maps, differential inclusions, stochastic variables, and new functions, system (44) becomes

$\dot{x}_{i}(t)$

$$
\begin{aligned}
\epsilon & -C_{i} x_{i}(t-\delta(t))+\sum_{j=1}^{m} \operatorname{co}\left[a_{j i}\left(x_{i}(t)\right)\right] f_{j}\left(y_{j}(t)\right) \\
& +\mu(t) \sum_{j=1}^{m} \operatorname{co}\left[b_{j i}\left(x_{i}(t)\right)\right] f_{j}\left(y_{j}\left(t-\tau_{1}(t)\right)\right) \\
& +(1-\mu(t)) \sum_{j=1}^{m} \operatorname{co}\left[b_{j i}\left(x_{i}(t)\right)\right] f_{j}\left(y_{j}\left(t-\tau_{2}(t)\right)\right) \\
& +\sum_{j=1}^{m} \operatorname{co}\left[k_{j i}\left(x_{i}(t)\right)\right] \int_{t-\alpha(t)}^{t} f(y(s)) d s+u_{i}(t),
\end{aligned}
$$

$\dot{y}_{j}(t)$

$$
\begin{aligned}
\epsilon & -D_{j} y_{j}(t-\rho(t))+\sum_{i=1}^{n} \mathrm{co}\left[e_{i j}\left(y_{j}(t)\right)\right] g_{i}\left(x_{i}(t)\right) \\
& +\omega(t) \sum_{i=1}^{n} \operatorname{co}\left[h_{i j}\left(y_{j}(t)\right)\right] g_{i}\left(x_{i}\left(t-d_{1}(t)\right)\right) \\
& +(1-\omega(t)) \sum_{i=1}^{n} \operatorname{co}\left[h_{i j}\left(y_{j}(t)\right)\right] g_{i}\left(x_{i}\left(t-d_{2}(t)\right)\right) \\
& +\sum_{i=1}^{n} \operatorname{co}\left[l_{i j}\left(y_{j}(t)\right)\right] \int_{t-\beta(t)}^{t} g(x(s)) d s+v_{j}(t) ;
\end{aligned}
$$

equivalently,

$$
\begin{aligned}
\dot{x}(t) & \\
= & -C x(t-\delta(t))+A(x(t)) f(y(t)) \\
& +\mu_{0} B(x(t)) f\left(y\left(t-\tau_{1}(t)\right)\right) \\
& +\left(1-\mu_{0}\right) B(x(t)) f\left(y\left(t-\tau_{2}(t)\right)\right) \\
& +\left(\mu(t)-\mu_{0}\right) \\
& \times B(x(t))\left(f\left(y\left(t-\tau_{1}(t)\right)\right)-f\left(y\left(t-\tau_{2}(t)\right)\right)\right) \\
& +K(x(t)) \int_{t-\alpha(t)}^{t} f(y(s)) d s+u(t),
\end{aligned}
$$

$\dot{y}(t)$

$$
\begin{aligned}
= & -D y(t-\rho(t))+E(y(t)) g(x(t)) \\
& +\omega_{0} H(y(t)) g\left(x\left(t-d_{1}(t)\right)\right) \\
& +\left(1-\omega_{0}\right) H(y(t)) g\left(x\left(t-d_{2}(t)\right)\right) \\
& +\left(\omega(t)-\omega_{0}\right)
\end{aligned}
$$

$$
\begin{aligned}
& \times H(y(t))\left(g\left(x\left(t-d_{1}(t)\right)\right)-g\left(x\left(t-d_{2}(t)\right)\right)\right) \\
& +L(y(t)) \int_{t-\beta(t)}^{t} g(x(s)) d s+v(t) .
\end{aligned}
$$

For presentation convenience, we denote

$$
\begin{gathered}
K=\max \left\{\left|\underline{k}_{j i}\right|,\left|\bar{k}_{j i}\right|\right\}, \\
L=\max \left\{\left|\underline{-i j}_{i j}\right|,\left|\overline{\bar{l}}_{i j}\right|\right\} .
\end{gathered}
$$

Theorem 9. Under Assumptions 1-4, system (50) is passive, if there exist any appropriately dimensional matrices $M_{m}(m=$ $1,2, \ldots, 4), W_{w}(w=1,2, \ldots, 6)$, a scalar $\gamma>0$, and symmetric positive definite matrices $R_{i}(i=1,2), Q_{j}(j=$ $1,2, \ldots, 6), O_{o}(o=1,2, \ldots, 6), P_{k}(k=1,2, \ldots, 8), Z_{l}(l=$ $1,2, \ldots, 4)$, and $N_{n}(n=1,2)$, such that

$$
\begin{aligned}
& \Omega_{i, j}=\left(\Omega_{i, j}\right)_{18 \times 18}<0, \\
& \Phi_{i, j}=\left(\Phi_{i, j}\right)_{18 \times 18}<0,
\end{aligned}
$$

where

$$
\begin{aligned}
\Omega_{1,1}= & -R_{1} C-C^{T} R_{1}+Q_{1}+Q_{2}+Q_{3}+Q_{4}+\delta^{2} P_{4} \\
& -P_{1}-P_{2}-2 Z_{1}-2 Z_{2}-2 \varsigma^{-} W_{1} \varsigma^{+}, \\
\Omega_{1,2}= & -M_{1} C+\left(1-\delta_{0}\right)^{2} P_{1}, \\
\Omega_{1,6}= & P_{2}, \\
\Omega_{1,8}= & -M_{1}, \\
\Omega_{1,9}= & R_{1} A+M_{1} A+W_{1}\left(\varsigma^{-}+\varsigma^{+}\right), \\
\Omega_{1,10}= & \mu_{0}\left(R_{1}+M_{1}\right) B, \\
\Omega_{1,11}= & \left(1-\mu_{0}\right)\left(M_{1}+R_{1}\right) B \\
\Omega_{1,14}= & C^{T} R_{1} C, \\
\Omega_{1,15}= & \frac{2}{\tau_{1}} Z_{1}, \\
\Omega_{1,16}= & \frac{2}{\tau_{2}-\tau_{1}} Z_{2}, \\
\Omega_{1,17}= & M_{1} K+R_{1} K \\
\Omega_{1,18}= & R_{1}+M_{1}, \\
\Omega_{2,2}= & -\left(1-\delta_{0}\right) Q_{1}-\left(1-\delta_{0}\right)^{2} P_{1}, \\
\Omega_{2,8}= & -M_{2} C, \\
\Omega_{3,3}= & -Q_{2}, \\
\Omega_{4,4}= & -\left(1-\mu_{1}\right) Q_{3}-2 \varsigma^{-} W_{2} \varsigma^{+}, \\
\Omega_{4,10}= & -\left(1-\mu_{1}\right) Q_{3}+W_{2}\left(\varsigma^{-}+\varsigma^{+}\right), \\
\Omega_{5,5}= & -\left(1-\mu_{2}\right) Q_{5}-2 \varsigma^{-} W_{3} \varsigma^{+}, \\
&
\end{aligned}
$$




$$
\begin{aligned}
& \Omega_{5,11}=-\left(1-\mu_{2}\right) Q_{5}+W_{3}\left(\varsigma^{-}+\varsigma^{+}\right), \\
& \Phi_{1,2}=-M_{3} C+\left(1-\rho_{0}\right)^{2} P_{5}, \\
& \Omega_{6,6}=Q_{5}+Q_{6}-Q_{4}-P_{2}-P_{3} \text {, } \\
& \Phi_{1,6}=P_{6} \text {, } \\
& \Omega_{6,7}=P_{3} \text {, } \\
& \Phi_{1,8}=-M_{3} \text {, } \\
& \Omega_{6,12}=Q_{5}+Q_{6}-Q_{4} \text {, } \\
& \Omega_{7,7}=-Q_{6}-P_{3} \text {, } \\
& \Omega_{7,13}=-Q_{6}, \\
& \Omega_{8,8}=\delta^{2} P_{1}+\tau_{1}^{2} P_{2}+\left(\tau_{2}-\tau_{1}\right)^{2} P_{3}+\frac{\tau_{1}^{2}}{2} Z_{1} \\
& +\frac{\tau_{2}^{2}-\tau_{1}^{2}}{2} Z_{2}-2 M_{2}, \\
& \Phi_{1,9}=R_{2} A+M_{3} A+W_{4}\left(\sigma^{-}+\sigma^{+}\right), \\
& \Phi_{1,10}=\omega_{0}\left(R_{2}+M_{3}\right) B, \\
& \Phi_{1,11}=\left(1-\omega_{0}\right)\left(M_{3}+R_{2}\right) B, \\
& \Phi_{1,14}=C^{T} R_{2} C \text {, } \\
& \Phi_{1,15}=\frac{2}{d_{1}} Z_{3}, \\
& \Omega_{8,9}=M_{2} A \text {, } \\
& \Omega_{8,10}=\mu_{0} M_{2} B, \\
& \Omega_{8,11}=\left(1-\mu_{0}\right) M_{2} B \text {, } \\
& \Omega_{8,17}=M_{2} K \text {, } \\
& \Omega_{8,18}=M_{2} \text {, } \\
& \Omega_{9,9}=-2 W_{1}+\alpha^{2} N_{1}, \\
& \Omega_{9,14}=-C^{T} R_{1} A \text {, } \\
& \Omega_{9,18}=-I, \\
& \Omega_{10,10}=-\left(1-\mu_{1}\right) Q_{3}-2 W_{2} \text {, } \\
& \Omega_{10,14}=-\mu_{0} C^{T} R_{1} B, \\
& \Omega_{11,11}=-\left(1-\mu_{2}\right) Q_{5}-2 W_{3} \text {, } \\
& \Omega_{11,14}=-\left(1-\mu_{0}\right) C^{T} R_{1} B, \\
& \Omega_{12,12}=Q_{5}+Q_{6}-Q_{4} \text {, } \\
& \Omega_{13,13}=-Q_{6} \text {, } \\
& \Omega_{14,14}=-P_{4} \text {, } \\
& \Omega_{14,17}=-C^{T} R_{1} K \text {, } \\
& \Omega_{14,18}=-C^{T} R_{1} \text {, } \\
& \Omega_{15,15}=-\frac{2}{\tau_{1}^{2}} Z_{1} \text {, } \\
& \Omega_{16,16}=-\frac{2}{\left(\tau_{2}-\tau_{1}\right)^{2}} Z_{2}, \\
& \Omega_{17,17}=-N_{1} \text {, } \\
& \Omega_{18,18}=-\gamma I \text {, } \\
& \Phi_{1,1}=-R_{2} C-C^{T} R_{2}+O_{1}+O_{2}+O_{3}+O_{4}+\rho^{2} P_{8} \\
& -P_{5}-P_{6}-2 Z_{3}-2 Z_{4}-2 \sigma^{-} W_{4} \sigma^{+} \text {, } \\
& \Phi_{1,16}=\frac{2}{d_{2}-d_{1}} Z_{4}, \\
& \Phi_{1,17}=M_{3} K+R_{2} K \text {, } \\
& \Phi_{1,18}=R_{2}+M_{3} \text {, } \\
& \Phi_{2,2}=-\left(1-\rho_{0}\right) O_{1}-\left(1-\rho_{0}\right)^{2} P_{5}, \\
& \Phi_{2,8}=-M_{4} C, \\
& \Phi_{3,3}=-\mathrm{O}_{2}, \\
& \Phi_{4,4}=-\left(1-\omega_{1}\right) O_{3}-2 \sigma^{-} W_{5} \sigma^{+}, \\
& \Phi_{4,10}=-\left(1-\omega_{1}\right) O_{3}+W_{5}\left(\sigma^{-}+\sigma^{+}\right), \\
& \Phi_{5,5}=-\left(1-\omega_{2}\right) O_{5}-2 \sigma^{-} W_{6} \sigma^{+}, \\
& \Phi_{5,11}=-\left(1-\omega_{2}\right) O_{5}+W_{6}\left(\sigma^{-}+\sigma^{+}\right) \text {, } \\
& \Phi_{6,6}=O_{5}+O_{6}-O_{4}-P_{6}-P_{7} \text {, } \\
& \Phi_{6,7}=P_{7}, \\
& \Phi_{6,12}=O_{5}+O_{6}-O_{4} \text {, } \\
& \Phi_{7,7}=-\mathrm{O}_{6}-P_{7} \text {, } \\
& \Phi_{7,13}=-O_{6} \text {, } \\
& \Phi_{8,8}=\rho^{2} P_{5}+d_{1}^{2} P_{6}+\left(d_{2}-d_{1}\right)^{2} P_{7}+\frac{d_{1}^{2}}{2} Z_{3} \\
& +\frac{d_{2}^{2}-d_{1}^{2}}{2} Z_{4}-2 M_{4}, \\
& \Phi_{8,9}=M_{4} A, \\
& \Phi_{8,10}=\omega_{0} M_{4} B, \\
& \Phi_{8,11}=\left(1-\omega_{0}\right) M_{4} B \text {, } \\
& \Phi_{8,17}=M_{4} K \text {, } \\
& \Phi_{8,18}=M_{4} \text {, } \\
& \Phi_{9,9}=-2 W_{4}+\alpha^{2} N_{2}, \\
& \Phi_{9,14}=-C^{T} R_{2} A \text {, }
\end{aligned}
$$




$$
\begin{aligned}
& \Phi_{9,18}=-I, \\
& \Phi_{10,10}=-\left(1-\omega_{1}\right) O_{3}-2 W_{5}, \\
& \Phi_{10,14}=-\omega_{0} C^{T} R_{2} B, \\
& \Phi_{11,11}=-\left(1-\omega_{2}\right) O_{5}-2 W_{6}, \\
& \Phi_{11,14}=-\left(1-\omega_{0}\right) C^{T} R_{2} B, \\
& \Phi_{12,12}=O_{5}+O_{6}-O_{4}, \\
& \Phi_{13,13}=-O_{6}, \\
& \Phi_{14,14}=-P_{8}, \\
& \Phi_{14,17}=-D^{T} R_{2} L, \\
& \Phi_{14,18}=-C^{T} R_{2}, \\
& \Phi_{15,15}=-\frac{2}{d_{1}^{2}} Z_{3}, \\
& \Phi_{16,16}=-\frac{2}{\left(d_{2}-d_{1}\right)^{2}} Z_{4}, \\
& \Phi_{17,17}=-N_{2}, \\
& \Phi_{18,18}=-\gamma I .
\end{aligned}
$$

Proof. Consider LKF candidate as follows:

$$
V(t)=\sum_{i=1}^{10} V_{i}(t)
$$

where

$$
\begin{gathered}
V_{1}(t)=\left(x(t)-C \int_{t-\delta(t)}^{t} x(s) d s\right)^{T} \\
\cdot R_{1}\left(x(t)-C \int_{t-\delta(t)}^{t} x(s) d s\right), \\
V_{2}(t)=\left(y(t)-D \int_{t-\rho(t)}^{t} y(s) d s\right)^{T} \\
\cdot R_{2}\left(y(t)-D \int_{t-\rho(t)}^{t} y(s) d s\right), \\
V_{3}(t)=\int_{t-\delta(t)}^{t} x^{T}(s) Q_{1} x(s) d s \\
+\int_{t-\delta}^{t} x^{T}(s) Q_{2} x(s) d s \\
+\int_{t-\tau_{1}(t)}^{t}\left[\begin{array}{c}
x(s) \\
f(y(s))
\end{array}\right]^{T} Q_{3}\left[\begin{array}{c}
x(s) \\
f(y(s))
\end{array}\right] d s \\
+\int_{t-\tau_{1}}^{t}\left[\begin{array}{c}
x(s) \\
f(y(s))
\end{array}\right]^{T} Q_{4}\left[\begin{array}{c}
x(s) \\
f(y(s))
\end{array}\right] d s
\end{gathered}
$$

$$
\begin{aligned}
& +\int_{t-\tau_{2}(t)}^{t-\tau_{1}}\left[\begin{array}{c}
x(s) \\
f(y(s))
\end{array}\right]^{T} Q_{5}\left[\begin{array}{c}
x(s) \\
f(y(s))
\end{array}\right] d s \\
& +\int_{t-\tau_{2}}^{t-\tau_{1}}\left[\begin{array}{c}
x(s) \\
f(y(s))
\end{array}\right]^{T} Q_{6}\left[\begin{array}{c}
x(s) \\
f(y(s))
\end{array}\right] d s, \\
& V_{4}(t)=\int_{t-\rho(t)}^{t} y^{T}(s) O_{1} y(s) d s \\
& +\int_{t-\rho}^{t} y^{T}(s) O_{2} y(s) d s \\
& +\int_{t-d_{1}(t)}^{t}\left[\begin{array}{c}
y(s) \\
g(x(s))
\end{array}\right]^{T} O_{3}\left[\begin{array}{c}
y(s) \\
g(x(s))
\end{array}\right] d s \\
& +\int_{t-d_{1}}^{t}\left[\begin{array}{c}
y(s) \\
g(x(s))
\end{array}\right]^{T} O_{4}\left[\begin{array}{c}
y(s) \\
g(x(s))
\end{array}\right] d s \\
& +\int_{t-d_{2}(t)}^{t-d_{1}}\left[\begin{array}{c}
y(s) \\
g(x(s))
\end{array}\right]^{T} O_{5}\left[\begin{array}{c}
y(s) \\
g(x(s))
\end{array}\right] d s \\
& +\int_{t-d_{2}}^{t-d_{1}}\left[\begin{array}{c}
y(s) \\
g(x(s))
\end{array}\right]^{T} O_{6}\left[\begin{array}{c}
y(s) \\
g(x(s))
\end{array}\right] d s, \\
& V_{5}(t)=\delta \int_{-\delta}^{0} \int_{t+\theta}^{t} \dot{x}^{T}(s) P_{1} \dot{x}(s) d s d \theta \\
& +\tau_{1} \int_{-\tau_{1}}^{0} \int_{t+\theta}^{t} \dot{x}^{T}(s) P_{2} \dot{x}(s) d s d \theta+\left(\tau_{2}-\tau_{1}\right) \\
& \cdot \int_{-\tau_{2}}^{-\tau_{1}} \int_{t+\theta}^{t} \dot{x}^{T}(s) P_{3} \dot{x}(s) d s d \theta \\
& +\delta \int_{-\delta}^{0} \int_{t+\theta}^{t} x^{T}(s) P_{4} x(s) d s d \theta, \\
& V_{6}(t)=\rho \int_{-\rho}^{0} \int_{t+\theta}^{t} \dot{y}^{T}(s) P_{5} \dot{y}(s) d s d \theta \\
& +d_{1} \int_{-d_{1}}^{0} \int_{t+\theta}^{t} \dot{y}^{T}(s) P_{6} \dot{y}(s) d s d \theta+\left(d_{2}-d_{1}\right) \\
& \cdot \int_{-d_{2}}^{-d_{1}} \int_{t+\theta}^{t} \dot{y}^{T}(s) P_{7} \dot{y}(s) d s d \theta \\
& +\rho \int_{-\delta}^{0} \int_{t+\theta}^{t} y^{T}(s) P_{8} y(s) d s d \theta \\
& V_{7}(t)=\int_{-\tau_{1}}^{0} \int_{\theta}^{0} \int_{t+\lambda}^{t} \dot{x}^{T}(s) Z_{1} \dot{x}(s) d s d \lambda d \theta \\
& +\int_{-\tau_{2}}^{-\tau_{1}} \int_{\theta}^{0} \int_{t+\lambda}^{t} \dot{x}^{T}(s) Z_{2} \dot{x}(s) d s d \lambda d \theta, \\
& V_{8}(t)=\int_{-d_{1}}^{0} \int_{\theta}^{0} \int_{t+\lambda}^{t} \dot{y}^{T}(s) Z_{3} \dot{y}(s) d s d \lambda d \theta \\
& +\int_{-d_{2}}^{-d_{1}} \int_{\theta}^{0} \int_{t+\lambda}^{t} \dot{y}^{T}(s) Z_{4} \dot{y}(s) d s d \lambda d \theta,
\end{aligned}
$$




$$
\begin{aligned}
& V_{9}(t)=\alpha \int_{-\alpha}^{0} \int_{t+\theta}^{t} f^{T}(y(s)) N_{1} f(y(s)) d s d \theta, \\
& V_{10}(t)=\beta \int_{-\beta}^{0} \int_{t+\theta}^{t} g^{T}(x(s)) N_{2} g(x(s)) d s d \theta .
\end{aligned}
$$

Then, we define the infinitesimal generator $\mathscr{L}$ of $V(t)$ as

$$
\mathscr{L} V(t)=\lim _{\Delta \rightarrow 0^{+}} \frac{1}{\Delta}\left\{\mathbb{E}\left(\frac{V(t+\Delta)}{t}\right)-V(t)\right\} .
$$

Taking the mathematical expectation of $V(t)$, we get

$$
\begin{aligned}
& \mathbb{E}\left\{\mathscr{L} V_{1}(t)\right\}=\mathbb{E}\left\{2\left(x(t)-C \int_{t-\delta(t)}^{t} x(s) d s\right)^{T} R_{1}\right. \\
& \times\left(-C x(t)+A(x(t)) f(y(t))+\mu_{0} B(x(t))\right. \\
& \times f\left(y\left(t-\tau_{1}(t)\right)\right)+\left(1-\mu_{0}\right) B(x(t)) \\
& \cdot f\left(y\left(t-\tau_{2}(t)\right)\right)+\left(\mu(t)-\mu_{0}\right) B(x(t)) \\
& \cdot\left(f\left(y\left(t-\tau_{1}(t)\right)\right)-f\left(y\left(t-\tau_{2}(t)\right)\right)\right)+K(x(t)) \\
& \left.\left.\cdot \int_{t-\alpha(t)}^{t} f(y(s)) d s+u(t)\right)\right\} \leq \mathbb{E}\{2(x(t) \\
& \left.-C \int_{t-\delta(t)}^{t} x(s) d s\right)^{T} R_{1} \times(-C x(t)+A f(y(t)) \\
& +\mu_{0} B f\left(y\left(t-\tau_{1}(t)\right)\right)+\left(1-\mu_{0}\right) \\
& \cdot B f\left(y\left(t-\tau_{2}(t)\right)\right)+K \int_{t-\alpha(t)}^{t} f(y(s)) d s \\
& +u(t))\},
\end{aligned}
$$$$
\mathbb{E}\left\{\mathscr{L} V_{2}(t)\right\}=\mathbb{E}\left\{2\left(y(t)-D \int_{t-\rho(t)}^{t} y(s) d s\right)^{T} R_{2}\right.
$$$$
\times\left(-D y(t)+E(y(t)) g(x(t))+\omega_{0} H(y(t))\right.
$$$$
\times g\left(x\left(t-d_{1}(t)\right)\right)+\left(1-\omega_{0}\right) H(y(t))
$$$$
\cdot g\left(x\left(t-d_{2}(t)\right)\right)+\left(\omega(t)-\omega_{0}\right) H(y(t))
$$$$
\cdot\left(g\left(x\left(t-d_{1}(t)\right)\right)-g\left(x\left(t-d_{2}(t)\right)\right)\right)+L(y(t))
$$$$
\left.\left.\cdot \int_{t-\beta(t)}^{t} g(x(s)) d s+v(t)\right)\right\} \leq \mathbb{E}\{2(y(t)
$$$$
\left.-D \int_{t-\rho(t)}^{t} y(s) d s\right)^{T} R_{2} \times(-D y(t)+E g(x(t))
$$$$
+\omega_{0} H g\left(x\left(t-d_{1}(t)\right)\right)+\left(1-\omega_{0}\right)
$$$$
\left.\left.\cdot H g\left(x\left(t-d_{2}(t)\right)\right)+L \int_{t-\beta(t)}^{t} g(x(s)) d s+v(t)\right)\right\} \text {. }
$$

$$
\begin{aligned}
& \text { According to Assumptions } 3 \text { and 4, we get } \\
& \mathbb{E}\left\{\mathscr{L} V_{3}(t)\right\}=\mathbb{E}\left\{x^{T}(t)\left(Q_{1}+Q_{2}+Q_{3}+Q_{4}\right) x(t)\right. \\
& -\left(1-\delta_{0}\right) x^{T}(t-\delta(t)) Q_{1} x(t-\delta(t)) \\
& -x^{T}(t-\delta) Q_{2} x(t-\delta)-\left(1-\mu_{1}\right) \\
& \cdot\left[\begin{array}{c}
x\left(t-\tau_{1}(t)\right) \\
f\left(y\left(t-\tau_{1}(t)\right)\right)
\end{array}\right]^{T} Q_{3}\left[\begin{array}{c}
x\left(t-\tau_{1}(t)\right) \\
f\left(y\left(t-\tau_{1}(t)\right)\right)
\end{array}\right] \\
& -\left(1-\mu_{2}\right)\left[\begin{array}{c}
x\left(t-\tau_{2}(t)\right) \\
f\left(y\left(t-\tau_{2}(t)\right)\right)
\end{array}\right]^{T} \\
& \cdot Q_{5}\left[\begin{array}{c}
x\left(t-\tau_{2}(t)\right) \\
f\left(y\left(t-\tau_{2}(t)\right)\right)
\end{array}\right]+\left[\begin{array}{c}
x\left(t-\tau_{1}\right) \\
f\left(y\left(t-\tau_{1}\right)\right)
\end{array}\right]^{T} \\
& \cdot\left(Q_{5}+Q_{6}-Q_{4}\right)\left[\begin{array}{c}
x\left(t-\tau_{1}\right) \\
f\left(y\left(t-\tau_{1}\right)\right)
\end{array}\right] \\
& \left.+\left[\begin{array}{c}
x\left(t-\tau_{2}\right) \\
f\left(y\left(t-\tau_{2}\right)\right)
\end{array}\right]^{T} Q_{6}\left[\begin{array}{c}
x\left(t-\tau_{2}\right) \\
f\left(y\left(t-\tau_{2}\right)\right)
\end{array}\right]\right\}, \\
& \mathbb{E}\left\{\mathscr{L} V_{4}(t)\right\}=\mathbb{E}\left\{y^{T}(t)\left(O_{1}+O_{2}+O_{3}+O_{4}\right) y(t)\right. \\
& -\left(1-\rho_{0}\right) y^{T}(t-\rho(t)) O_{1} y(t-\rho(t)) \\
& -y^{T}(t-\rho) O_{2} y(t-\rho)-\left(1-\omega_{1}\right) \\
& \cdot\left[\begin{array}{c}
y\left(t-d_{1}(t)\right) \\
g\left(x\left(t-d_{1}(t)\right)\right)
\end{array}\right]^{T} O_{3}\left[\begin{array}{c}
y\left(t-d_{1}(t)\right) \\
g\left(x\left(t-d_{1}(t)\right)\right)
\end{array}\right] \\
& -\left(1-\omega_{2}\right)\left[\begin{array}{c}
x\left(t-d_{2}(t)\right) \\
g\left(x\left(t-d_{2}(t)\right)\right)
\end{array}\right]^{T} \\
& \cdot O_{5}\left[\begin{array}{c}
y\left(t-d_{2}(t)\right) \\
g\left(x\left(t-d_{2}(t)\right)\right)
\end{array}\right]+\left[\begin{array}{c}
x\left(t-d_{1}\right) \\
g\left(x\left(t-d_{1}\right)\right)
\end{array}\right]^{T} \\
& \cdot\left(O_{5}+O_{6}-O_{4}\right)\left[\begin{array}{c}
y\left(t-d_{1}\right) \\
g\left(x\left(t-d_{1}\right)\right)
\end{array}\right] \\
& \left.+\left[\begin{array}{c}
y\left(t-d_{2}\right) \\
g\left(x\left(t-d_{2}\right)\right)
\end{array}\right]^{T} O_{6}\left[\begin{array}{c}
y\left(t-d_{2}\right) \\
g\left(x\left(t-d_{2}\right)\right)
\end{array}\right]\right\} .
\end{aligned}
$$

It is obvious that

$$
\begin{aligned}
\mathbb{E} & \left\{\mathscr{L} V_{5}(t)\right\} \\
& =\mathbb{E}\left\{\dot{x}^{T}(t)\left[\delta^{2} P_{1}+\tau_{1}^{2} P_{2}+\left(\tau_{2}-\tau_{1}\right)^{2} P_{3}\right] \dot{x}(t)\right. \\
& +\delta^{2} x^{T}(t) P_{4} x(t)-\delta \int_{t-\delta}^{t} \dot{x}^{T}(s) P_{1} \dot{x}(s) d s
\end{aligned}
$$




$$
\begin{aligned}
& -\tau_{1} \int_{t-\tau_{1}}^{t} \dot{x}^{T}(s) P_{2} \dot{x}(s) d s \\
& -\left(\tau_{2}-\tau_{1}\right) \int_{t-\tau_{2}}^{t-\tau_{1}} \dot{x}^{T}(s) P_{3} \dot{x}(s) d s \\
& \left.-\delta \int_{t-\delta}^{t} x^{T}(s) P_{4} x(s) d s\right\}, \\
& \mathbb{E}\left\{\mathscr{L} V_{6}(t)\right\} \\
& \quad=\mathbb{E}\left\{\dot{y}^{T}(t)\left[\rho^{2} P_{5}+d_{1}^{2} P_{6}+\left(d_{2}-d_{1}\right)^{2} P_{7}\right] \dot{y}(t)\right. \\
& +\rho^{2} y^{T}(t) P_{8} y(t)-\rho \int_{t-\rho}^{t} \dot{y}^{T}(s) P_{5} \dot{y}(s) d s \\
& -d_{1} \int_{t-d_{1}}^{t} \dot{y}^{T}(s) P_{6} \dot{y}(s) d s \\
& -\left(d_{2}-d_{1}\right) \int_{t-d_{2}}^{t-d_{1}} \dot{y}^{T}(s) P_{7} \dot{y}(s) d s \\
& \left.-\rho \int_{t-\rho}^{t} y^{T}(s) P_{8} y(s) d s\right\} .
\end{aligned}
$$

Using Lemma 7, we have

$$
\begin{aligned}
& -\delta \int_{t-\delta}^{t} \dot{x}^{T}(s) P_{1} \dot{x}(s) d s \\
& \leq-\left(\int_{t-\delta(t)}^{t} \dot{x}(s) d s\right)^{T} P_{1}\left(\int_{t-\delta(t)}^{t} \dot{x}(s) d s\right) \\
& =-\left[x(t)-\left(1-\delta_{0}\right) x(t-\delta)\right]^{T} P_{1} \\
& \quad \times\left[x(t)-\left(1-\delta_{0}\right) x(t-\delta)\right], \\
& -\delta \int_{t-\delta}^{t} x^{T}(s) P_{4} x(s) d s \\
& \leq-\left(\int_{t-\delta(t)}^{t} x(s) d s\right)^{T} P_{4}\left(\int_{t-\delta(t)}^{t} x(s) d s\right) .
\end{aligned}
$$

Similarly, we have

$$
\begin{aligned}
& -\rho \int_{t-\rho}^{t} \dot{y}^{T}(s) P_{4} \dot{y}(s) d s \\
& \leq-\left(\int_{t-\rho(t)}^{t} \dot{y}(s) d s\right)^{T} P_{4}\left(\int_{t-\rho(t)}^{t} \dot{y}(s) d s\right) \\
& =-\left[y(t)-\left(1-\rho_{0}\right) y(t-\rho)\right]^{T} P_{4} \\
& \quad \times\left[y(t)-\left(1-\rho_{0}\right) y(t-\rho)\right], \\
& -\rho \int_{t-\rho}^{t} y^{T}(s) P_{8} y(s) d s \\
& \leq-\left(\int_{t-\rho(t)}^{t} y(s) d s\right)^{T} P_{8}\left(\int_{t-\rho(t)}^{t} y(s) d s\right) .
\end{aligned}
$$

Then, the same as (32)-(35), we can obtain $\mathbb{E}\left\{\mathscr{L} V_{7}(t)\right\}$ and $\mathbb{E}\left\{\mathscr{L} V_{8}(t)\right\}$.

Moreover,

$$
\begin{gathered}
\mathbb{E}\left\{\mathscr{L} V_{9}(t)\right\}=\mathbb{E}\left\{\alpha^{2} f^{T}(y(t)) N_{1} f(y(t))\right. \\
\left.-\alpha \int_{t-\alpha}^{t} f^{T}(y(s)) N_{1} f(y(s)) d s\right\}, \\
\mathbb{E}\left\{\mathscr{L} V_{10}(t)\right\}=\mathbb{E}\left\{\beta^{2} g^{T}(x(t)) N_{2} g(x(t))\right. \\
\left.-\beta \int_{t-\beta}^{t} g^{T}(x(s)) N_{2} g(x(s)) d s\right\} .
\end{gathered}
$$

Also, we get

$$
\begin{aligned}
& -\alpha \int_{t-\alpha}^{t} f^{T}(y(s)) N_{1} f(y(s)) d s \\
& \leq-\left(\int_{t-\alpha(t)}^{t} f(y(s)) d s\right)^{T} N_{1}\left(\int_{t-\alpha(t)}^{t} f(y(s)) d s\right), \\
& -\beta \int_{t-\beta}^{t} g^{T}(x(s)) N_{2} g(x(s)) d s \\
& \leq-\left(\int_{t-\beta(t)}^{t} g(x(s)) d s\right)^{T} N_{2}\left(\int_{t-\beta(t)}^{t} g(x(s)) d s\right) .
\end{aligned}
$$

Now, we add the following inequations with any matrices $M_{1}, M_{2}, M_{3}$, and $M_{4}$ of appropriate dimensions to derive a less conservative criterion:

$$
\begin{aligned}
0 \leq & 2\left(x^{T}(t) M_{1}+\dot{x}^{T}(t) M_{2}\right)[-\dot{x}-C x(t-\delta(t)) \\
& +A f(y(t))+\mu_{0} B f\left(y\left(t-\tau_{1}(t)\right)\right) \\
& +\left(1-\mu_{0}\right) B f\left(y\left(t-\tau_{2}(t)\right)\right) \\
& \left.+K \int_{t-\alpha(t)}^{t} f(y(s)) d s+u(t)\right], \\
0 \leq & 2\left(y^{T}(t) M_{3}+\dot{y}^{T}(t) M_{4}\right)[-\dot{y}-D y(t-\rho(t)) \\
& +E g(x(t))+\omega_{0} H g\left(x\left(t-d_{1}(t)\right)\right) \\
& +\left(1-\omega_{0}\right) H g\left(x\left(t-d_{2}(t)\right)\right) \\
& \left.+L \int_{t-\beta(t)}^{t} g(x(s)) d s+v(t)\right] .
\end{aligned}
$$

Define

$$
\begin{aligned}
& \xi(t)=\left\{\begin{array}{llll}
x^{T}(t) & x^{T}(t-\delta(t)) & x^{T}(t-\delta) & x^{T}\left(t-\tau_{1}(t)\right)
\end{array}\right. \\
& x^{T}\left(t-\tau_{2}(t)\right) \quad x^{T}\left(t-\tau_{1}\right) \quad x^{T}\left(t-\tau_{2}\right) \quad \dot{x}(t)
\end{aligned}
$$




$$
\begin{aligned}
& f^{T}(y(t)) \quad f^{T}\left(y\left(t-\tau_{1}(t)\right)\right) \quad f^{T}\left(y\left(t-\tau_{2}(t)\right)\right) \\
& f^{T}\left(y\left(t-\tau_{1}\right)\right) f^{T}\left(y\left(t-\tau_{2}\right)\right) \int_{t-\delta(t)}^{t} x^{T}(s) d s \\
& \left.\int_{t-\tau_{1}}^{t} x^{T}(s) d s \int_{t-\tau_{2}}^{t-\tau_{1}} x^{T}(s) d s \int_{t-\alpha(t)}^{t} f^{T}(y(s)) d s u^{T}(t)\right\}, \\
& \zeta(t)=\left\{\begin{array}{llll}
y^{T}(t) & y^{T}(t-\rho(t)) & y^{T}(t-\rho) & y^{T}\left(t-d_{1}(t)\right)
\end{array}\right. \\
& y^{T}\left(t-d_{2}(t)\right) \quad y^{T}\left(t-d_{1}\right) \quad y^{T}\left(t-d_{2}\right) \quad \dot{y}(t) \\
& g^{T}(x(t)) \quad g^{T}\left(x\left(t-d_{1}(t)\right)\right) \quad g^{T}\left(x\left(t-d_{2}(t)\right)\right) \\
& g^{T}\left(x\left(t-d_{1}\right)\right) g^{T}\left(x\left(t-d_{2}\right)\right) \int_{t-\rho(t)}^{t} y^{T}(s) d s \\
& \left.\int_{t-d_{1}}^{t} y^{T}(s) d s \int_{t-d_{2}}^{t-d_{1}} y^{T}(s) d s \int_{t-\beta(t)}^{t} g^{T}(x(s)) d s \quad v^{T}(t)\right\} .
\end{aligned}
$$

Combining (40) and (58)-(66), we have

$$
\begin{aligned}
\mathbb{E} & \left\{\mathscr{L} V(t)-2\left[f^{T}(y(s)) g^{T}(x(s))\right]\left[\begin{array}{l}
u(s) \\
v(s)
\end{array}\right]\right. \\
& \left.-\gamma\left[u^{T}(t) v^{T}(t)\right]\left[\begin{array}{l}
u(t) \\
v(t)
\end{array}\right] d s\right\} \leq \mathbb{E}\left\{\xi^{T}(t) \Omega \xi(t)\right. \\
& \left.+\zeta^{T}(t) \Phi \zeta(t)\right\} .
\end{aligned}
$$

Thus, we conclude that if (68) holds, then

$$
\begin{aligned}
& \mathbb{E}\left\{\mathscr{L} V(t)-2\left[f^{T}(y(s)) g^{T}(x(s))\right]\left[\begin{array}{l}
u(s) \\
v(s)
\end{array}\right]\right. \\
& \left.-\gamma\left[\begin{array}{ll}
u^{T}(t) & v^{T}(t)
\end{array}\right]\left[\begin{array}{l}
u(t) \\
v(t)
\end{array}\right] d s\right\} \leq 0 .
\end{aligned}
$$

By integrating (69) about $t$ from 0 to $t_{z}$, we have

$$
\begin{aligned}
& 2 \mathbb{E} \int_{0}^{t_{z}}\left[f^{T}(y(s)) g^{T}(x(s))\right]\left[\begin{array}{l}
u(s) \\
v(s)
\end{array}\right] d s \\
& \geq \mathbb{E} V(t)-\mathbb{E} V(0)
\end{aligned}
$$

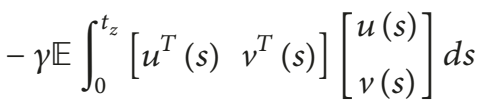

$$
\begin{aligned}
& \geq-\gamma \mathbb{E} \int_{0}^{t_{z}}\left[\begin{array}{lll}
u^{T}(s) & v^{T}(s)
\end{array}\right]\left[\begin{array}{l}
u(s) \\
v(s)
\end{array}\right] d s .
\end{aligned}
$$

Therefore, from Definition 5, we know that the MBAMNN is passive. This completes the proof.

Remark 10. In this paper, model (50) contains the leakage, probabilistic, and distributed time-varying delays. In particular, we treat some of these delays as a special case of some existing results.
Remark 11. In the corollary, we define leakage delays $\delta$ and $\rho$ as constant delays. According to the preceding conditions, we consider the MBAMNN in the following:

$\dot{x}(t)$

$$
\begin{aligned}
= & -C x(t-\delta)+A(x(t)) f(y(t)) \\
& +\mu_{0} B(x(t)) f\left(y\left(t-\tau_{1}(t)\right)\right) \\
& +\left(1-\mu_{0}\right) B(x(t)) f\left(y\left(t-\tau_{2}(t)\right)\right) \\
& +\left(\mu(t)-\mu_{0}\right) \\
& \times B(x(t))\left(f\left(y\left(t-\tau_{1}(t)\right)\right)-f\left(y\left(t-\tau_{2}(t)\right)\right)\right) \\
& +u(t)
\end{aligned}
$$

$\dot{y}(t)$

$$
\begin{aligned}
= & -D y(t-\rho)+E(y(t)) g(x(t)) \\
& +\omega_{0} H(y(t)) g\left(x\left(t-d_{1}(t)\right)\right) \\
& +\left(1-\omega_{0}\right) H(y(t)) g\left(x\left(t-d_{2}(t)\right)\right) \\
& +\left(\omega(t)-\omega_{0}\right) \\
& \times H(y(t))\left(g\left(x\left(t-d_{1}(t)\right)\right)-g\left(x\left(t-d_{2}(t)\right)\right)\right) \\
& +v(t) .
\end{aligned}
$$

Corollary 12. When Assumptions 1-3 hold, system (71) is passive, if there exist any appropriately dimensional matrices $M_{m}(m=1,2, \ldots, 4), W_{w}(w=1,2, \ldots, 6)$, a scalar $\gamma>0$, and symmetric positive definite matrices $R_{i}(i=1,2), Q_{j}(j=$ $2, \ldots, 6), O_{o}(o=2, \ldots, 6), P_{k}(k=1,2, \ldots, 8)$, and $Z_{l}(l=$ $1,2, \ldots, 4)$, such that

$$
\begin{aligned}
& \Omega_{i, j}=\left(\Omega_{i, j}\right)_{17 \times 17}<0, \\
& \Phi_{i, j}=\left(\Phi_{i, j}\right)_{17 \times 17}<0,
\end{aligned}
$$

where

$$
\begin{aligned}
\Omega_{1,1}= & -R_{1} C-C^{T} R_{1}+Q_{2}+Q_{3}+Q_{4}+\delta^{2} P_{4}-P_{1} \\
& -P_{2}-2 Z_{1}-2 Z_{2}-2 \varsigma^{-} W_{1} \varsigma^{+}, \\
\Omega_{1,2}= & P_{1}-M_{1} C \\
\Omega_{1,5}= & P_{2} \\
\Omega_{1,7}= & -M_{1}, \\
\Omega_{1,8}= & R_{1} A+M_{1} A+W_{1}\left(\varsigma^{-}+\varsigma^{+}\right), \\
\Omega_{1,9}= & \mu_{0}\left(R_{1}+M_{1}\right) B, \\
\Omega_{1,10}= & \left(1-\mu_{0}\right)\left(M_{1}+R_{1}\right) B \\
\Omega_{1,13}= & C^{T} R_{1} C \\
\Omega_{1,14}= & \frac{2}{\tau_{1}} Z_{1},
\end{aligned}
$$




$$
\begin{aligned}
& \Omega_{1,15}=\frac{2}{\tau_{2}-\tau_{1}} Z_{2}, \\
& \Omega_{14,14}=-\frac{2}{\tau_{1}^{2}} Z_{1}, \\
& \Omega_{1,16}=M_{1} K \text {, } \\
& \Omega_{1,17}=R_{1}+M_{1} \text {, } \\
& \Omega_{2,2}=-Q_{2}-P_{1} \text {, } \\
& \Omega_{2,7}=-M_{2} C \text {, } \\
& \Omega_{3,3}=-\left(1-\mu_{1}\right) Q_{3}-2 \varsigma^{-} W_{2} \varsigma^{+}, \\
& \Omega_{3,9}=-\left(1-\mu_{1}\right) Q_{3}+W_{2}\left(\varsigma^{-}+\varsigma^{+}\right) \text {, } \\
& \Omega_{4,4}=-\left(1-\mu_{2}\right) Q_{5}-2 \varsigma^{-} W_{3} \varsigma^{+}, \\
& \Omega_{4,10}=-\left(1-\mu_{2}\right) Q_{5}+W_{3}\left(\varsigma^{-}+\varsigma^{+}\right) \text {, } \\
& \Omega_{5,5}=Q_{5}+Q_{6}-Q_{4}-P_{2}-P_{3} \text {, } \\
& \Omega_{5,6}=P_{3} \text {, } \\
& \Omega_{5,11}=Q_{5}+Q_{6}-Q_{4} \text {, } \\
& \Omega_{6,6}=-Q_{6}-P_{3} \text {, } \\
& \Omega_{6,12}=-Q_{6} \text {, } \\
& \Omega_{7,7}=\delta^{2} P_{1}+\tau_{1}^{2} P_{2}+\left(\tau_{2}-\tau_{1}\right)^{2} P_{3}+\frac{\tau_{1}^{2}}{2} Z_{1} \\
& +\frac{\tau_{2}^{2}-\tau_{1}^{2}}{2} Z_{2}-2 M_{2} \\
& \Omega_{7,8}=M_{2} A \text {, } \\
& \Omega_{7,9}=\mu_{0} M_{2} B, \\
& \Omega_{7,10}=\left(1-\mu_{0}\right) M_{2} B, \\
& \Omega_{7,16}=M_{2} K \text {, } \\
& \Omega_{7,17}=M_{2} \text {, } \\
& \Omega_{8,8}=-2 W_{1}+\alpha^{2} N_{1}, \\
& \Omega_{8,13}=-C^{T} R_{1} A, \\
& \Omega_{8,17}=-I \text {, } \\
& \Omega_{9,9}=-\left(1-\mu_{1}\right) Q_{3}-2 W_{2}, \\
& \Omega_{9,13}=-\mu_{0} C^{T} R_{1} B, \\
& \Omega_{10,10}=-\left(1-\mu_{2}\right) Q_{5}-2 W_{3} \text {, } \\
& \Omega_{10,13}=-\left(1-\mu_{0}\right) C^{T} R_{1} B, \\
& \Omega_{11,11}=Q_{5}+Q_{6}-Q_{4} \text {, } \\
& \Omega_{12,12}=-Q_{6} \text {, } \\
& \Omega_{13,13}=-P_{4} \text {, } \\
& \Omega_{13,17}=-C^{T} R_{1} \text {, } \\
& \Omega_{15,15}=-\frac{2}{\left(\tau_{2}-\tau_{1}\right)^{2}} Z_{2}, \\
& \Omega_{16,16}=-N_{1} \text {, } \\
& \Omega_{17,17}=-\gamma I \text {, } \\
& \Phi_{1,1}=-R_{2} C-C^{T} R_{2}+O_{2}+O_{3}+O_{4}+\rho^{2} P_{8}-P_{5} \\
& -P_{6}-2 Z_{3}-2 Z_{4}-2 \sigma^{-} W_{4} \sigma^{+} \text {, } \\
& \Phi_{1,2}=P_{5}-M_{3} D \text {, } \\
& \Phi_{1,5}=P_{6} \text {, } \\
& \Phi_{1,7}=-M_{3} \text {, } \\
& \Phi_{1,8}=R_{2} A+M_{3} A+W_{4}\left(\sigma^{-}+\sigma^{+}\right), \\
& \Phi_{1,9}=\omega_{0}\left(R_{2}+M_{3}\right) B \text {, } \\
& \Phi_{1,10}=\left(1-\omega_{0}\right)\left(M_{3}+R_{2}\right) B \text {, } \\
& \Phi_{1,13}=C^{T} R_{2} C \text {, } \\
& \Phi_{1,14}=\frac{2}{d_{1}} Z_{3} \text {, } \\
& \Phi_{1,15}=\frac{2}{d_{2}-d_{1}} Z_{4} \text {, } \\
& \Phi_{1,16}=M_{3} K \text {, } \\
& \Phi_{1,17}=R_{2}+M_{3} \text {, } \\
& \Phi_{2,2}=-\mathrm{O}_{2}-P_{5} \text {, } \\
& \Phi_{2,7}=-M_{4} C \text {, } \\
& \Phi_{3,3}=-\left(1-\omega_{1}\right) O_{3}-2 \sigma^{-} W_{5} \sigma^{+}, \\
& \Phi_{3,9}=-\left(1-\omega_{1}\right) O_{3}+W_{5}\left(\sigma^{-}+\sigma^{+}\right), \\
& \Phi_{4,4}=-\left(1-\omega_{2}\right) O_{5}-2 \sigma^{-} W_{6} \sigma^{+}, \\
& \Phi_{4,10}=-\left(1-\omega_{2}\right) O_{5}+W_{6}\left(\sigma^{-}+\sigma^{+}\right) \text {, } \\
& \Phi_{5,5}=O_{5}+O_{6}-O_{4}-P_{6}-P_{7}, \\
& \Phi_{5,6}=P_{7} \text {, } \\
& \Phi_{5,11}=\mathrm{O}_{5}+\mathrm{O}_{6}-\mathrm{O}_{4} \text {, } \\
& \Phi_{6,6}=-\mathrm{O}_{6}-P_{7} \text {, } \\
& \Phi_{6,12}=-\mathrm{O}_{6} \text {, } \\
& \Phi_{7,7}=\rho^{2} P_{5}+d_{1}^{2} P_{6}+\left(d_{2}-d_{1}\right)^{2} P_{7}+\frac{d_{1}^{2}}{2} Z_{3} \\
& +\frac{d_{2}^{2}-d_{1}^{2}}{2} Z_{4}-2 M_{4}
\end{aligned}
$$




$$
\begin{aligned}
& \Phi_{7,8}=M_{4} A, \\
& \Phi_{7,9}=\omega_{0} M_{4} B, \\
& \Phi_{7,10}=\left(1-\omega_{0}\right) M_{4} B, \\
& \Phi_{7,16}=M_{4} K, \\
& \Phi_{7,17}=M_{4} \text {, } \\
& \Phi_{8,8}=-2 W_{4}+\alpha^{2} N_{2}, \\
& \Phi_{8,13}=-C^{T} R_{2} A, \\
& \Phi_{8,17}=-I \text {, } \\
& \Phi_{9,9}=-\left(1-\omega_{1}\right) O_{3}-2 W_{5}, \\
& \Phi_{9,13}=-\omega_{0} C^{T} R_{2} B, \\
& \Phi_{10,10}=-\left(1-\omega_{2}\right) O_{5}-2 W_{6} \text {, } \\
& \Phi_{10,13}=-\left(1-\omega_{0}\right) C^{T} R_{2} B, \\
& \Phi_{11,11}=O_{5}+O_{6}-O_{4}, \\
& \Phi_{12,12}=-\mathrm{O}_{6} \text {, } \\
& \Phi_{13,13}=-P_{8} \text {, } \\
& \Phi_{13,17}=-C^{T} R_{2} \text {, } \\
& \Phi_{14,14}=-\frac{2}{d_{1}^{2}} Z_{3}, \\
& \Phi_{15,15}=-\frac{2}{\left(d_{2}-d_{1}\right)^{2}} Z_{4} \text {, } \\
& \Phi_{16,16}=-N_{2} \text {, } \\
& \Phi_{17,17}=-\gamma I \text {. }
\end{aligned}
$$

Remark 13. In numerical simulations, we use the models of MBAMNNs with mixed time-varying delays; the models that we used are novel. In the existing papers, we can see that the most of the papers are about passivity analysis of memristive single-layer neural networks and there are few results about MBAMNNs [17-19]. MBAMNNs are a class of two-layer systems which can realize heteroassociation memory and autoassociation memory; hence, the MBAMNNs can be able to imitate the human brain better, and the limitation of traditional neural networks can be broken. The LKFs that we designed include double and triple integral terms, and using some effective methods, we can obtain several conditions about MBAMNNs with leakage, probabilistic, and distributed time-varying delays. Hence, our research results are less conservative than the existing ones in the models and the treatment methods $[19,21]$. In particular, these delays are all taken into consideration separately. Thus, our paper not only improves the models but also considers a variety of timevarying delays [18-21].

\section{Numerical Simulation}

In this section, we provide several numerical examples to illustrate the usefulness of our stability results.

Example 1. We consider a 2-dimensional MBAMNN of (16) with the parameters as follows:

$$
\begin{aligned}
& a_{11}\left(x_{1}(t)\right)= \begin{cases}0.3, & \left|x_{1}(t)\right|>\Phi_{1}, \\
0.7, & \left|x_{1}(t)\right| \leq \Phi_{1},\end{cases} \\
& a_{12}\left(x_{1}(t)\right)= \begin{cases}0.4, & \left|x_{1}(t)\right|>\Phi_{1}, \\
0.5, & \left|x_{1}(t)\right| \leq \Phi_{1},\end{cases} \\
& a_{21}\left(x_{2}(t)\right)= \begin{cases}-0.5, & \left|x_{2}(t)\right|>\Phi_{2} \\
-0.6, & \left|x_{2}(t)\right| \leq \Phi_{2}\end{cases} \\
& a_{22}\left(x_{2}(t)\right)= \begin{cases}-0.76, & \left|x_{2}(t)\right|>\Phi_{2} \\
-0.7, & \left|x_{2}(t)\right| \leq \Phi_{2}\end{cases} \\
& b_{11}\left(x_{1}(t)\right)= \begin{cases}0.33, & \left|x_{1}(t)\right|>\Phi_{1}, \\
0.3, & \left|x_{1}(t)\right| \leq \Phi_{1},\end{cases} \\
& b_{12}\left(x_{1}(t)\right)= \begin{cases}0.25, & \left|x_{1}(t)\right|>\Phi_{1} \\
0.2, & \left|x_{1}(t)\right| \leq \Phi_{1}\end{cases} \\
& b_{21}\left(x_{2}(t)\right)= \begin{cases}0.6, & \left|x_{2}(t)\right|>\Phi_{2} \\
0.56, & \left|x_{2}(t)\right| \leq \Phi_{2}\end{cases} \\
& b_{22}\left(x_{2}(t)\right)= \begin{cases}0.63, & \left|x_{2}(t)\right|>\Phi_{2} \\
0.6, & \left|x_{2}(t)\right| \leq \Phi_{2}\end{cases} \\
& e_{11}\left(y_{1}(t)\right)= \begin{cases}1.3, & \left|y_{1}(t)\right|>\Psi_{1} \\
0.4, & \left|y_{1}(t)\right| \leq \Psi_{1}\end{cases} \\
& e_{12}\left(y_{1}(t)\right)= \begin{cases}1.28, & \left|y_{1}(t)\right|>\Psi_{1} \\
0.38, & \left|y_{1}(t)\right| \leq \Psi_{1}\end{cases} \\
& e_{21}\left(y_{2}(t)\right)= \begin{cases}-1.05, & \left|y_{2}(t)\right|>\Psi_{2}, \\
-1.1, & \left|y_{2}(t)\right| \leq \Psi_{2},\end{cases} \\
& e_{22}\left(y_{2}(t)\right)= \begin{cases}-0.88, & \left|y_{2}(t)\right|>\Psi_{2}, \\
-0.8, & \left|y_{2}(t)\right| \leq \Psi_{2},\end{cases} \\
& h_{11}\left(y_{1}(t)\right)= \begin{cases}0.38, & \left|y_{1}(t)\right|>\Psi_{1}, \\
0.4, & \left|y_{1}(t)\right| \leq \Psi_{1},\end{cases}
\end{aligned}
$$




$$
\begin{aligned}
& h_{12}\left(y_{1}(t)\right)= \begin{cases}0.25, & \left|y_{1}(t)\right|>\Psi_{1}, \\
0.2, & \left|y_{1}(t)\right| \leq \Psi_{1},\end{cases} \\
& h_{21}\left(y_{2}(t)\right)= \begin{cases}0.5, & \left|y_{2}(t)\right|>\Psi_{2}, \\
0.87, & \left|y_{2}(t)\right| \leq \Psi_{2},\end{cases} \\
& h_{22}\left(y_{2}(t)\right)= \begin{cases}0.38, & \left|y_{2}(t)\right|>\Psi_{2}, \\
-0.5, & \left|y_{2}(t)\right| \leq \Psi_{2} .\end{cases}
\end{aligned}
$$

Here, we take the activation functions as $f_{i}(s)=g_{i}(s)=$ $\tanh (s), i=1,2$. Then, let $\tau_{1}=0.5, \tau_{2}=0.6, \mu_{1}=0.3, \mu_{2}=$ $0.5, \mu_{0}=0.4, d_{1}=1.5, d_{2}=2, \omega_{1}=0.6, \omega_{2}=0.8$, and $\omega_{0}=0.3$. Probabilistic time-varying delays $\tau_{1}(t)=0.7+$ $0.2 \sin (t), \tau_{2}(t)=0.65+0.6 \cos (0.5 t), d_{1}(t)=0.4+0.4 \sin (t)$, and $d_{2}(t)=0.3+0.3 \cos (t)$. According to Assumption 1, we verify that $\varsigma^{-}=\sigma^{-}=0$, and $\varsigma^{+}=\sigma^{+}=1$. Then, there exist the matrices as follows:

$$
\begin{aligned}
C & =\left[\begin{array}{cc}
1.52 & 0 \\
0 & 1.33
\end{array}\right], \\
D & =\left[\begin{array}{cc}
1.21 & 0 \\
0 & 1.76
\end{array}\right], \\
A & =\left[\begin{array}{cc}
0.7 & 0.5 \\
-0.6 & -0.76
\end{array}\right], \\
B & =\left[\begin{array}{cc}
0.33 & 0.25 \\
0.6 & 0.63
\end{array}\right], \\
E & =\left[\begin{array}{cc}
1.3 & 1.28 \\
-1.1 & -0.88
\end{array}\right], \\
H & =\left[\begin{array}{cc}
0.4 & 0.25 \\
0.87 & -0.5
\end{array}\right] .
\end{aligned}
$$

Figure 3 shows the state trajectories of system (16); it is actually unstable. By solving the LMIs (21), we get the following feasible solutions:

$$
\begin{aligned}
R_{1} & =\left[\begin{array}{ll}
10.3478 & 2.3731 \\
2.3731 & 9.8579
\end{array}\right], \\
Q_{3} & =\left[\begin{array}{ll}
3.1634 & 0.6423 \\
0.6423 & 3.7317
\end{array}\right], \\
Q_{4} & =\left[\begin{array}{ll}
7.1504 & 1.4750 \\
1.4750 & 8.3565
\end{array}\right], \\
Q_{5} & =\left[\begin{array}{ll}
4.0425 & 1.1230 \\
1.1230 & 4.5925
\end{array}\right], \\
Q_{6} & =\left[\begin{array}{ll}
1.9672 & 0.2093 \\
0.2093 & 2.3559
\end{array}\right],
\end{aligned}
$$

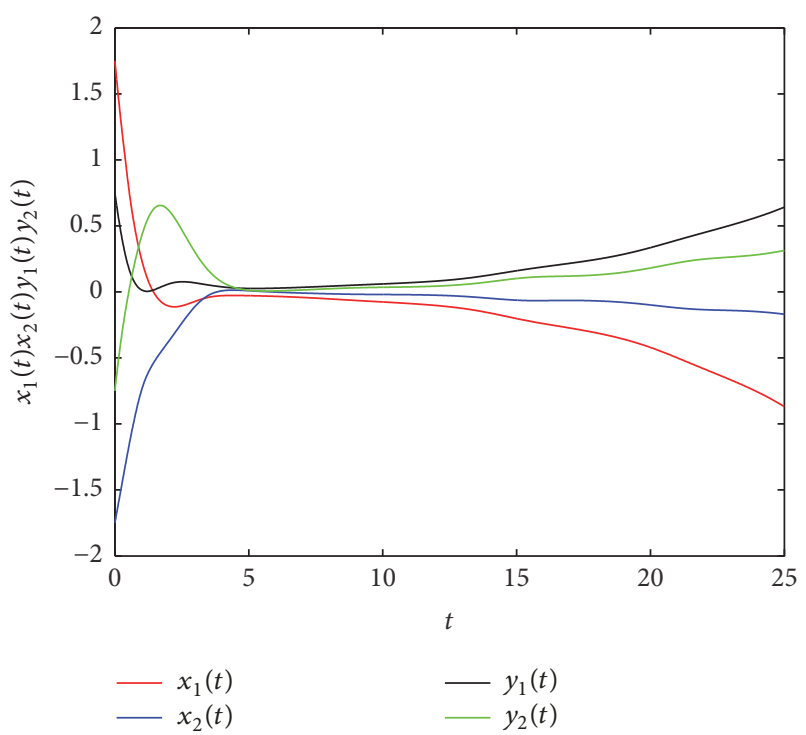

FIgURE 3: State trajectories of system (16).

$$
\begin{aligned}
& P_{2}=\left[\begin{array}{ll}
4.3159 & 0.1023 \\
0.1023 & 4.0132
\end{array}\right], \\
& P_{3}=\left[\begin{array}{cc}
4.7189 & -0.0015 \\
-0.0015 & 4.6924
\end{array}\right] \text {, } \\
& Z_{1}=\left[\begin{array}{ll}
0.3110 & 0.0541 \\
0.0541 & 0.3985
\end{array}\right], \\
& Z_{2}=\left[\begin{array}{cc}
0.0457 & -0.0004 \\
-0.0004 & 0.0450
\end{array}\right], \\
& R_{2}=\left[\begin{array}{cc}
14.0110 & 3.7932 \\
3.7932 & 14.2218
\end{array}\right] \text {, } \\
& O_{3}=\left[\begin{array}{ll}
3.6595 & 0.9958 \\
0.9958 & 4.4985
\end{array}\right] \text {, } \\
& O_{4}=\left[\begin{array}{ll}
8.0128 & 2.0885 \\
2.0885 & 9.9776
\end{array}\right] \text {, } \\
& O_{5}=\left[\begin{array}{ll}
5.3912 & 2.2361 \\
2.2361 & 6.2646
\end{array}\right] \text {, } \\
& O_{6}=\left[\begin{array}{cc}
1.6776 & -0.0893 \\
-0.0893 & 2.3607
\end{array}\right] \text {, } \\
& P_{6}=\left[\begin{array}{ll}
7.4849 & 0.1076 \\
0.1076 & 7.7770
\end{array}\right] \text {, } \\
& P_{7}=\left[\begin{array}{cc}
8.7529 & -0.0353 \\
-0.0353 & 8.8750
\end{array}\right] \text {, }
\end{aligned}
$$




$$
\begin{aligned}
& Z_{3}=\left[\begin{array}{cc}
0.3312 & -0.0060 \\
-0.0060 & 0.4389
\end{array}\right], \\
& Z_{4}=\left[\begin{array}{ll}
0.2262 & 0.0004 \\
0.0004 & 0.2189
\end{array}\right] \text {, } \\
& M_{1}=\left[\begin{array}{ll}
1.7488 & 0.1600 \\
0.1600 & 1.7944
\end{array}\right] \text {, } \\
& M_{2}=\left[\begin{array}{ll}
4.3667 & 0.4139 \\
0.4139 & 4.0400
\end{array}\right] \text {, } \\
& W_{1}=\left[\begin{array}{ll}
5.4082 & 3.0213 \\
3.0213 & 6.4308
\end{array}\right], \\
& W_{2}=\left[\begin{array}{ll}
3.5446 & 0.5053 \\
0.5053 & 3.7780
\end{array}\right], \\
& W_{3}=\left[\begin{array}{ll}
3.5391 & 0.8050 \\
0.8050 & 3.7611
\end{array}\right], \\
& M_{3}=\left[\begin{array}{ll}
2.0055 & 0.2579 \\
0.2579 & 2.2772
\end{array}\right] \text {, } \\
& M_{4}=\left[\begin{array}{ll}
8.1716 & 1.6020 \\
1.6020 & 7.4034
\end{array}\right] \text {, } \\
& W_{4}=\left[\begin{array}{ll}
7.9401 & 4.1641 \\
4.1641 & 9.2674
\end{array}\right] \text {, } \\
& W_{5}=\left[\begin{array}{ll}
5.0707 & 1.2361 \\
1.2361 & 5.9315
\end{array}\right] \text {, } \\
& W_{6}=\left[\begin{array}{ll}
5.3825 & 2.0405 \\
2.0405 & 6.1309
\end{array}\right], \\
& \gamma=20.4722 \text {. }
\end{aligned}
$$

Using the feasible solution, we obtain the state trajectories which are described in Figure 4, and we can see that both $x(t)$ and $y(t)$ are converging to the zero point; therefore, the proved MBAMNN is internally stable. Thus, it is globally passive.

Example 2. Consider a 2-dimensional MBAMNN of (50) with the parameters as follows.

Here, we take the activation functions as $f_{i}(s)=g_{i}(s)=$ $\tanh (s), i=1,2$. Then, let $\delta=0.2, \delta_{0}=0.2, \tau_{1}=0.6, \tau_{2}=$ $1, \mu_{1}=0.3, \mu_{2}=0.5, \mu_{0}=0.4, \alpha=0.1, \rho=0.18, \rho_{0}=$ $0.15, d_{1}=1.5, d_{2}=2, \omega_{1}=0.6, \omega_{2}=0.8, \omega_{0}=0.3$, and $\beta=$ 0.2 . Leakage time-varying delays $\delta(t)=0.5+0.3 \sin (t)$ and $\rho(t)=0.4+0.5 \cos (t)$; probabilistic time-varying delays $\tau_{1}(t)=0.4+0.3 \sin (t), \tau_{2}(t)=0.5+0.5 \cos (0.5 t), d_{1}(t)=$ $0.3+0.3 \sin (t)$, and $d_{2}(t)=0.1+0.5 \cos (t)$; distributed

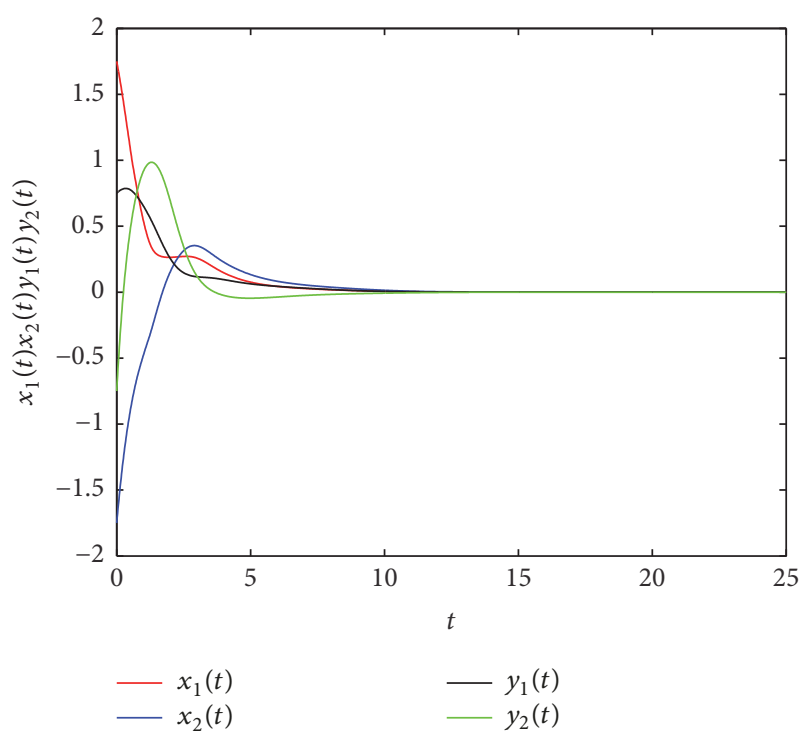

FIGURE 4: State trajectories of system (16).

time-varying delays $\alpha(t)=\beta(t)=0.5+0.5 \cos (t)$. After changing parameters, there exist the matrices

$$
\begin{aligned}
C & =\left[\begin{array}{cc}
1.52 & 0 \\
0 & 1.33
\end{array}\right], \\
D & =\left[\begin{array}{cc}
1.21 & 0 \\
0 & 1.76
\end{array}\right], \\
A & =\left[\begin{array}{cc}
0.3 & 0.4 \\
-0.6 & -0.76
\end{array}\right], \\
B & =\left[\begin{array}{cc}
0.33 & 0.25 \\
0.6 & 0.63
\end{array}\right], \\
E & =\left[\begin{array}{cc}
1.3 & 1.28 \\
-1.1 & -0.88
\end{array}\right], \\
H & =\left[\begin{array}{cc}
0.4 & 0.25 \\
0.87 & -0.5
\end{array}\right], \\
K & =\left[\begin{array}{cc}
0.12 & 0 \\
0 & -0.1
\end{array}\right], \\
L & =\left[\begin{array}{cc}
-0.1 & 0 \\
0 & -0.06
\end{array}\right] .
\end{aligned}
$$

From the state trajectories which are depicted in Figure 5, we find that system (50) is actually unstable. By solving the LMIs (52), we have the following feasible solutions:

$$
R_{1}=\left[\begin{array}{cc}
7.0452 & 2.9361 \\
2.9361 & 11.6063
\end{array}\right]
$$




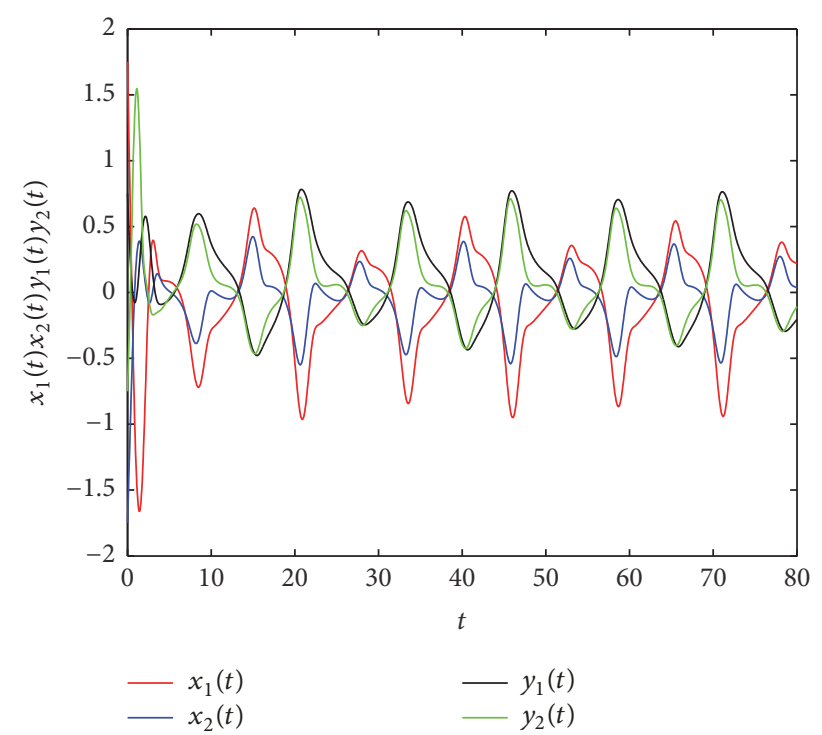

FIGURE 5: State trajectories of system (50).

$$
\begin{aligned}
& Q_{1}=\left[\begin{array}{cc}
6.5971 & -1.6049 \\
-1.6049 & 9.2078
\end{array}\right] \text {, } \\
& Q_{2}=\left[\begin{array}{ll}
0.5551 & 0.4601 \\
0.4601 & 3.5493
\end{array}\right], \\
& Q_{3}=\left[\begin{array}{ll}
1.7184 & 1.0171 \\
1.0171 & 5.9298
\end{array}\right], \\
& Q_{4}=\left[\begin{array}{ll}
3.5521 & 2.1946 \\
2.1946 & 13.1443
\end{array}\right], \\
& Q_{5}=\left[\begin{array}{ll}
2.6799 & 1.5175 \\
1.5175 & 7.6941
\end{array}\right] \text {, } \\
& Q_{6}=\left[\begin{array}{ll}
0.5770 & 0.4402 \\
0.4402 & 3.5446
\end{array}\right], \\
& P_{1}=\left[\begin{array}{cc}
5.6725 & -11.6662 \\
-11.6662 & 76.6622
\end{array}\right] \text {, } \\
& P_{2}=\left[\begin{array}{ll}
0.0858 & 0.0272 \\
0.0272 & 1.0798
\end{array}\right], \\
& P_{3}=\left[\begin{array}{ll}
0.1919 & 0.0517 \\
0.0517 & 2.2235
\end{array}\right], \\
& P_{4}=\left[\begin{array}{cc}
478.9177 & 72.1940 \\
72.1940 & 122.7031
\end{array}\right] \text {, } \\
& Z_{1}=\left[\begin{array}{ll}
0.0835 & 0.0516 \\
0.0516 & 0.6378
\end{array}\right] \text {, }
\end{aligned}
$$

$$
\begin{aligned}
& Z_{2}=\left[\begin{array}{cc}
0.1446 & -0.0612 \\
-0.0612 & 1.8780
\end{array}\right] \text {, } \\
& M_{1}=\left[\begin{array}{ll}
1.1710 & 1.1366 \\
1.1366 & 2.7731
\end{array}\right] \text {, } \\
& M_{2}=\left[\begin{array}{ll}
0.5725 & 0.0760 \\
0.0760 & 5.8435
\end{array}\right] \text {, } \\
& W_{1}=\left[\begin{array}{cc}
2.9231 & 3.9208 \\
3.9208 & 15.5396
\end{array}\right] \text {, } \\
& W_{2}=\left[\begin{array}{ll}
2.5516 & 1.3400 \\
1.3400 & 8.4107
\end{array}\right] \text {, } \\
& W_{3}=\left[\begin{array}{ll}
2.7557 & 1.4738 \\
1.4738 & 7.8878
\end{array}\right] \text {, } \\
& N_{1}=\left[\begin{array}{cc}
20.6455 & 0.1674 \\
0.1674 & 25.5954
\end{array}\right] \text {, } \\
& R_{2}=\left[\begin{array}{ll}
22.1627 & 10.5483 \\
10.5483 & 21.6007
\end{array}\right] \text {, } \\
& O_{1}=\left[\begin{array}{rr}
29.6665 & -0.0928 \\
-0.0928 & 17.2449
\end{array}\right] \text {, } \\
& \mathrm{O}_{2}=\left[\begin{array}{ll}
2.1311 & 0.8588 \\
0.8588 & 3.0198
\end{array}\right] \text {, } \\
& O_{3}=\left[\begin{array}{ll}
6.9160 & 1.2013 \\
1.2013 & 7.3828
\end{array}\right] \text {, } \\
& O_{4}=\left[\begin{array}{cc}
20.7004 & 2.6570 \\
2.6570 & 20.3705
\end{array}\right] \text {, } \\
& O_{5}=\left[\begin{array}{cc}
17.4326 & 1.3252 \\
1.3252 & 15.6910
\end{array}\right] \text {, } \\
& O_{6}=\left[\begin{array}{ll}
2.1603 & 0.8746 \\
0.8746 & 3.0879
\end{array}\right] \text {, } \\
& P_{5}=\left[\begin{array}{cc}
77.8766 & -12.7230 \\
-12.7230 & 176.5013
\end{array}\right] \text {, } \\
& P_{6}=\left[\begin{array}{ll}
0.1260 & 0.0457 \\
0.0457 & 0.1924
\end{array}\right] \text {, } \\
& P_{7}=\left[\begin{array}{ll}
0.8705 & 0.3558 \\
0.3558 & 1.3869
\end{array}\right] \text {, } \\
& P_{8}=\left[\begin{array}{ll}
860.5842 & 235.3961 \\
235.3961 & 447.6840
\end{array}\right] \text {, } \\
& Z_{3}=\left[\begin{array}{ll}
0.2328 & 0.0901 \\
0.0901 & 0.3687
\end{array}\right] \text {, }
\end{aligned}
$$




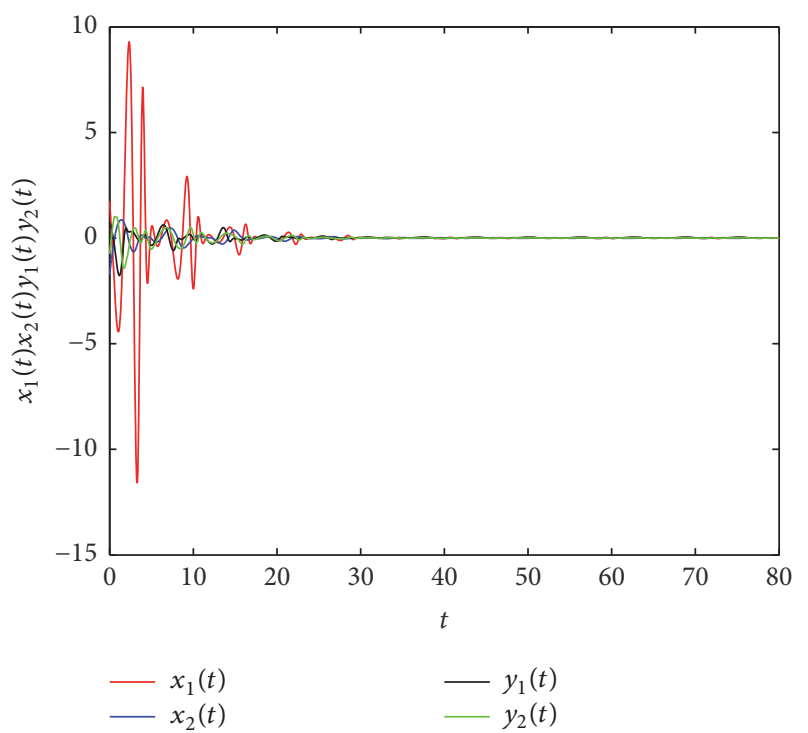

FIGURE 6: State trajectories of system (50).

$$
\begin{aligned}
Z_{4} & =\left[\begin{array}{ll}
0.3230 & 0.1292 \\
0.1292 & 0.5292
\end{array}\right], \\
M_{3} & =\left[\begin{array}{ll}
5.2095 & 2.3987 \\
2.3987 & 3.2962
\end{array}\right], \\
M_{4} & =\left[\begin{array}{ll}
5.1434 & 1.3833 \\
1.3833 & 8.8319
\end{array}\right], \\
W_{4} & =\left[\begin{array}{ll}
25.3297 & 14.2601 \\
14.2601 & 34.2731
\end{array}\right], \\
W_{5} & =\left[\begin{array}{ll}
5.9791 & 1.1879 \\
1.1879 & 6.6651
\end{array}\right], \\
W_{6} & =\left[\begin{array}{ll}
7.1161 & 0.6066 \\
0.6066 & 6.5388
\end{array}\right], \\
N_{2} & =\left[\begin{array}{ll}
40.5252 & 2.2784 \\
2.2784 & 38.2262
\end{array}\right], \\
\gamma & =50.2752 .
\end{aligned}
$$

Using the feasible solution, we obtain the state trajectories in Figure 6, and we find that both $x(t)$ and $y(t)$ are converging to the zero point; thus, the proved MBAMNN is internally stable. Therefore, it is globally passive.

Example 3. Consider a 2-dimensional MBAMNN of (71) with the parameters as follows.

Here, we take the activation functions as $f_{i}(s)=g_{i}(s)=$ $\tanh (s), i=1,2$. And let $\delta=0.3, \tau_{1}=0.2, \tau_{2}=0.3, \mu_{1}=$ $0.3, \mu_{2}=0.4, \mu_{0}=0.4, d_{1}=1.3, d_{2}=1.5, \omega_{1}=0.6, \omega_{2}=$

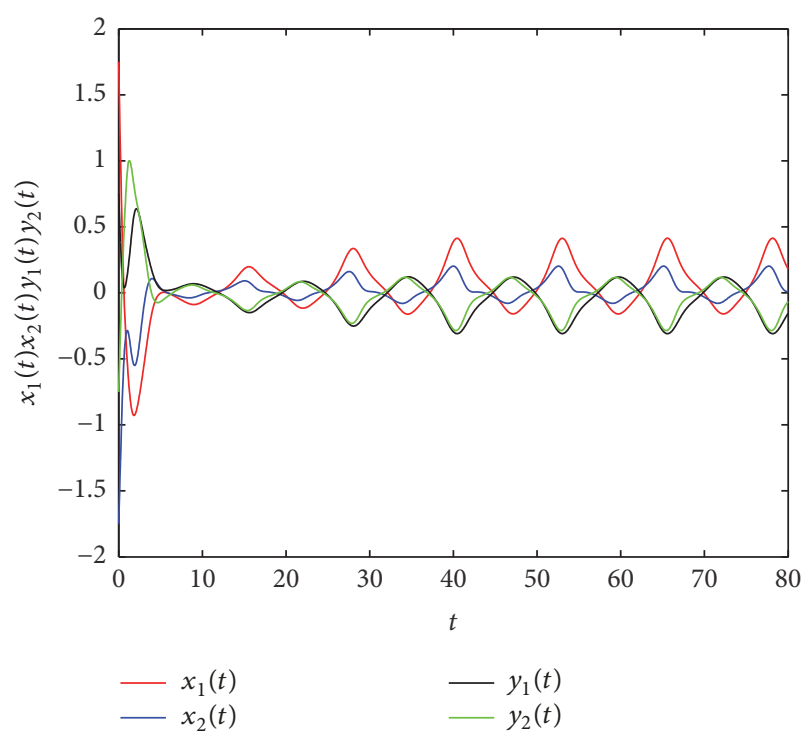

Figure 7: State trajectories of system (71).

0.8 , and $\omega_{0}=0.25$. Probabilistic time-varying delays $\tau_{1}(t)=$ $0.6+0.4 \sin (t), \tau_{2}(t)=0.8+0.5 \cos (0.5 t), d_{1}(t)=0.5+$ $0.3 \sin (t)$, and $d_{2}(t)=0.6+0.5 \cos (t)$. After changing parameters, there exist the matrices

$$
\begin{aligned}
& C=\left[\begin{array}{cc}
1.52 & 0 \\
0 & 1.33
\end{array}\right], \\
& D=\left[\begin{array}{cc}
1.21 & 0 \\
0 & 1.76
\end{array}\right], \\
& A=\left[\begin{array}{cc}
0.7 & 0.5 \\
-0.6 & -0.76
\end{array}\right], \\
& B=\left[\begin{array}{cc}
0.33 & 0.25 \\
0.6 & 0.63
\end{array}\right], \\
& E=\left[\begin{array}{cc}
1.3 & 1.28 \\
-1.1 & -0.88
\end{array}\right], \\
& H=\left[\begin{array}{cc}
0.4 & 0.25 \\
0.87 & -0.5
\end{array}\right] .
\end{aligned}
$$

Figure 7 shows the state trajectories of system (71); it is actually unstable. By solving the LMIs in Corollary 12, we obtain the following feasible solutions:

$$
\begin{aligned}
R_{1} & =\left[\begin{array}{ll}
59.3283 & 30.3806 \\
30.3806 & 56.1253
\end{array}\right], \\
Q_{2} & =\left[\begin{array}{ll}
11.2692 & 4.8104 \\
4.8104 & 13.1800
\end{array}\right],
\end{aligned}
$$




$$
\begin{aligned}
& Q_{3}=\left[\begin{array}{cc}
14.8695 & 8.8717 \\
8.8717 & 14.8525
\end{array}\right], \\
& Q_{4}=\left[\begin{array}{ll}
29.6331 & 17.8695 \\
17.8695 & 30.7251
\end{array}\right] \text {, } \\
& Q_{5}=\left[\begin{array}{ll}
21.2565 & 12.7666 \\
12.7666 & 19.9407
\end{array}\right] \text {, } \\
& Q_{6}=\left[\begin{array}{ll}
5.4645 & 3.2592 \\
3.2592 & 6.9941
\end{array}\right] \text {, } \\
& P_{1}=\left[\begin{array}{cc}
36.1361 & 6.0248 \\
6.0248 & 31.4429
\end{array}\right] \text {, } \\
& P_{2}=\left[\begin{array}{cc}
25.8085 & 3.8441 \\
3.8441 & 27.0796
\end{array}\right] \text {, } \\
& P_{3}=\left[\begin{array}{cc}
33.1166 & 1.3714 \\
1.3714 & 34.0596
\end{array}\right] \text {, } \\
& P_{4}=\left[\begin{array}{cc}
296.1210 & 93.8823 \\
93.8823 & 252.4336
\end{array}\right] \text {, } \\
& Z_{1}=\left[\begin{array}{ll}
0.1137 & 0.0919 \\
0.0919 & 0.1535
\end{array}\right] \text {, } \\
& Z_{2}=\left[\begin{array}{cc}
0.7613 & -0.1194 \\
-0.1194 & 0.7099
\end{array}\right], \\
& M_{1}=\left[\begin{array}{cc}
8.3925 & 0.6621 \\
0.6621 & 10.4709
\end{array}\right] \text {, } \\
& M_{2}=\left[\begin{array}{cc}
12.4316 & 0.3775 \\
0.3775 & 13.7920
\end{array}\right] \text {, } \\
& W_{1}=\left[\begin{array}{ll}
27.1266 & 17.2502 \\
17.2502 & 33.8015
\end{array}\right] \text {, } \\
& W_{2}=\left[\begin{array}{ll}
19.6366 & 10.3508 \\
10.3508 & 19.7140
\end{array}\right] \text {, } \\
& W_{3}=\left[\begin{array}{ll}
23.9080 & 13.1438 \\
13.1438 & 22.5256
\end{array}\right] \text {, } \\
& R_{2}=\left[\begin{array}{ll}
25.5707 & 20.3266 \\
20.3266 & 41.5693
\end{array}\right] \text {, } \\
& O_{2}=\left[\begin{array}{cc}
15.1779 & 4.9012 \\
4.9012 & 43.6250
\end{array}\right] \text {, } \\
& O_{3}=\left[\begin{array}{cc}
13.7722 & 0.9440 \\
0.9440 & 8.8489
\end{array}\right] \text {, }
\end{aligned}
$$$$
M_{4}=\left[\begin{array}{cc}
17.9661 & 1.5273 \\
1.5273 & 15.4807
\end{array}\right] \text {, }
$$$$
W_{4}=\left[\begin{array}{ll}
55.1410 & 44.6653 \\
44.6653 & 57.6623
\end{array}\right] \text {, }
$$$$
O_{6}=\left[\begin{array}{ll}
4.9903 & 1.5755 \\
1.5755 & 3.5372
\end{array}\right] \text {, }
$$$$
P_{5}=\left[\begin{array}{ll}
104.0967 & 34.3385 \\
34.3385 & 87.8965
\end{array}\right] \text {, }
$$$$
P_{6}=\left[\begin{array}{ll}
0.8159 & 0.3667 \\
0.3667 & 0.4678
\end{array}\right] \text {, }
$$$$
P_{7}=\left[\begin{array}{cc}
24.1326 & 8.8218 \\
8.8218 & 15.3127
\end{array}\right] \text {, }
$$$$
P_{8}=\left[\begin{array}{ll}
220.2420 & 251.8009 \\
251.8009 & 686.0176
\end{array}\right] \text {, }
$$$$
Z_{3}=\left[\begin{array}{ll}
2.2774 & 1.0053 \\
1.0053 & 1.2605
\end{array}\right] \text {, }
$$$$
Z_{4}=\left[\begin{array}{cc}
38.7115 & 4.7302 \\
4.7302 & 32.7023
\end{array}\right] \text {, }
$$$$
M_{3}=\left[\begin{array}{ll}
2.8959 & 0.6752 \\
0.6752 & 8.0316
\end{array}\right] \text {, }
$$$$
W_{5}=\left[\begin{array}{cc}
12.3093 & 1.3710 \\
1.3710 & 8.1296
\end{array}\right] \text {, }
$$$$
W_{6}=\left[\begin{array}{cc}
15.9416 & -1.5426 \\
-1.5426 & 9.4538
\end{array}\right] \text {, }
$$$$
O_{4}=\left[\begin{array}{rr}
46.4425 & -1.9760 \\
-1.9760 & 28.1517
\end{array}\right] \text {, }
$$$$
O_{5}=\left[\begin{array}{ll}
38.9043 & -4.3696 \\
-4.3696 & 22.8177
\end{array}\right] \text {, }
$$$$
\gamma=183.3912 \text {. }
$$

Using the feasible solution, we obtain the state trajectories in Figure 8, and we can see that both $x(t)$ and $y(t)$ are converging to the zero point; therefore, the proved MBAMNN is internally stable. Thus, it is globally passive.

\section{Conclusion}

We have studied the passivity problem of MBAMNNs with probabilistic and mixed time-varying delays in this paper. By introducing random variables with Bernoulli distribution, using some useful inequalities, and constructing appropriate 


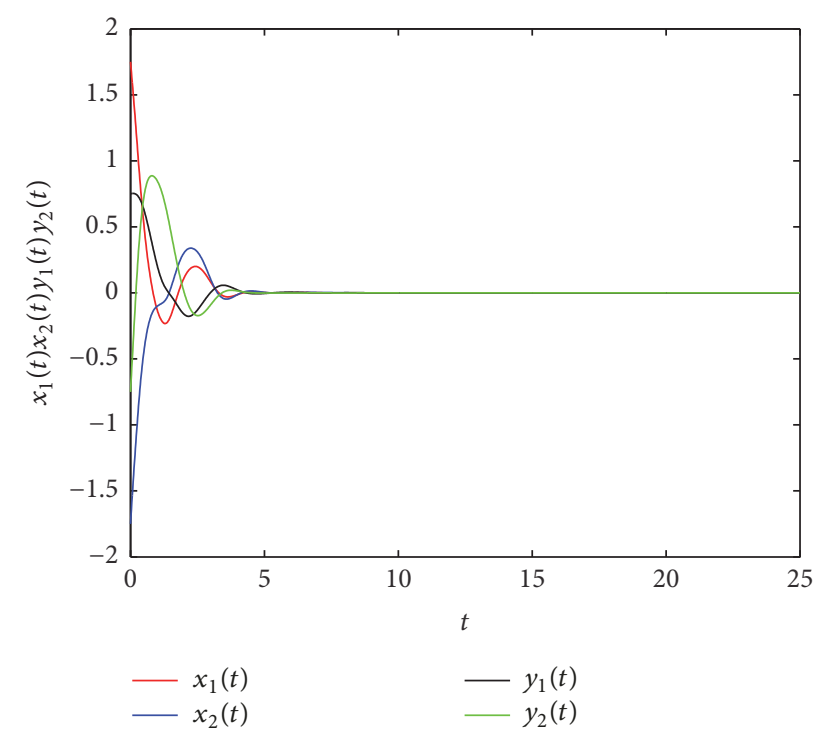

FIGURE 8: State trajectories of system (71).

LKFs, we have got new delay-dependent conditions in LMIs, which ensure the passivity criteria. Future work will focus on the passivity analysis of MBAMNNs with different types of time delays.

\section{Conflicts of Interest}

The authors declare that there are no conflicts of interest regarding the publication of this paper.

\section{Authors' Contributions}

Weiping Wang and Xiong Luo contributed equally to this work.

\section{Acknowledgments}

This work was supported by the National Key Research and Development Program of China under Grant 2017YFB0702300, the State Scholarship Fund of China Scholarship Council (CSC), the National Natural Science Foundation of China under Grants 61603032 and 61174103, the Fundamental Research Funds for the Central Universities under Grant 06500025, the National Key Technologies R\&D Program of China under Grant 2015BAK38B01, and the University of Science and Technology Beijing-National Taipei University of Technology Joint Research Program under Grant TW201705.

\section{References}

[1] B. Wang, J. Jian, and M. Jiang, "Global stability in Lagrange sense for BAM-type Cohen-Grossberg neural networks with time-varying delays," Systems Science \& Control Engineering, vol. 3, no. 1, pp. 1-7, 2015.
[2] Y. Song, M. Han, and J. Wei, "Stability and Hopf bifurcation analysis on a simplified BAM neural network with delays," Physica D: Nonlinear Phenomena, vol. 200, no. 3-4, pp. 185-204, 2005.

[3] Y. K. Li, L. Yang, and W. Q. Wu, "Anti-periodic solution for impulsive BAM neural networks with time-varying leakage delays on time scales," Neurocomputing, vol. 149, pp. 536-545, 2015.

[4] M. Yu, W. Wang, X. Luo, L. Liu, and M. Yuan, "Exponential antisynchronization control of stochastic memristive neural networks with mixed time-varying delays based on novel delaydependent or delay-independent adaptive controller," Mathematical Problems in Engineering, vol. 2017, Article ID 8314757, pp. 1-16, 2017.

[5] J. D. Cao and Y. Wan, "Matrix measure strategies for stability and synchronization of inertial BAM neural network with time delays," Neural Networks, vol. 53, pp. 165-172, 2014.

[6] Z. Cai and L. Huang, "Functional differential inclusions and dynamic behaviors for memristor-based BAM neural networks with time-varying delays," Communications in Nonlinear Science \& Numerical Simulation, vol. 19, no. 5, pp. 1279-1300, 2014.

[7] J. Qi, C. Li, and T. Huang, "Stability of inertial BAM neural network with time-varying delay via impulsive control," Neurocomputing, vol. 161, pp. 162-167, 2015.

[8] L. Zhang, S. P. University, and S. O. Science, “"Exponential stability of BAM neural network with time-varying delays," Journal of Baoshan University, vol. 31, no. 1, article 385, 396 pages, 2016.

[9] F. Wang, Y. Yang, X. Xu, and L. Li, "Global asymptotic stability of impulsive fractional-order BAM neural networks with time delay," Neural Computing \& Applications, vol. 30, no. 2, pp. 1-8, 2017.

[10] R. Zhang, D. Zeng, S. Zhong, and Y. Yu, "Event-triggered sampling control for stability and stabilization of memristive neural networks with communication delays," Applied Mathematics and Computation, vol. 310, pp. 57-74, 2017.

[11] X. Wang, C. Li, T. Huang, and S. Duan, "Global exponential stability of a class of memristive neural networks with timevarying delays," Neural Computing and Applications, vol. 24, no. 7-8, pp. 1707-1715, 2014.

[12] Z. Guo, J. Wang, and Z. Yan, "Global exponential synchronization of two memristor-based recurrent neural networks with time delays via static or dynamic coupling," Systems Man \& Cybernetics Systems IEEE Transactions, vol. 45, no. 2, 2014.

[13] W. Wang, L. Li, H. Peng, J. Kurths, J. Xiao, and Y. Yang, "Antisynchronization control of memristive neural networks with multiple proportional delays," Neural Processing Letters, vol. 43, no. 1, pp. 269-283, 2016.

[14] K. Mathiyalagan, J. H. Park, and R. Sakthivel, "Synchronization for delayed memristive BAM neural networks using impulsive control with random nonlinearities," Applied Mathematics and Computation, vol. 259, pp. 967-979, 2015.

[15] R. Sakthivel, R. Anbuvithya, K. Mathiyalagan, Y.-K. Ma, and P. Prakash, "Reliable anti-synchronization conditions for BAM memristive neural networks with different memductance functions," Applied Mathematics and Computation, vol. 275, pp. 213228, 2016.

[16] R. Anbuvithya, K. Mathiyalagan, R. Sakthivel, and P. Prakash, "Non-fragile synchronization of memristive BAM networks with random feedback gain fluctuations," Communications in Nonlinear Science and Numerical Simulation, vol. 29, no. 1-3, pp. 427-440, 2015. 
[17] M. Jiang, S. Wang, J. Mei, and Y. Shen, "Finite-time synchronization control of a class of memristor-based recurrent neural networks," Neural Networks, vol. 63, pp. 133-140, 2015.

[18] Z. Meng and Z. Xiang, "Passivity analysis of memristor-based recurrent neural networks with mixed time-varying delays," Neurocomputing, vol. 165, pp. 270-279, 2015.

[19] H. Li, H. Gao, and P. Shi, "New passivity analysis for neural networks with discrete and distributed delays," IEEE Transactions on Neural Networks and Learning Systems, vol. 21, no. 11, pp. 1842-1847, 2010.

[20] M. Syed Ali, R. Saravanakumar, and J. Cao, "New passivity criteria for memristor-based neutral-type stochastic BAM neural networks with mixed time-varying delays," Neurocomputing, vol. 171, pp. 1533-1547, 2016.

[21] J. Liu and R. Xu, "Passivity analysis and state estimation for a class of memristor-based neural networks with multiple proportional delays," Advances in Difference Equations, vol. 2017, article 34, 2017.

[22] R. Anbuvithya, K. Mathiyalagan, R. Sakthivel, and P. Prakash, "Passivity of memristor-based BAM neural networks with different memductance and uncertain delays," Cognitive Neurodynamics, vol. 10, no. 4, pp. 339-351, 2016.

[23] R. Zhang, D. Zeng, and S. Zhong, "Novel master-slave synchronization criteria of chaotic Lur'e systems with time delays using sampled-data control," Journal of The Franklin Institute, vol. 354, no. 12, pp. 4930-4954, 2017.

[24] H. Wu, X. Zhang, R. Li, and R. Yao, "Adaptive antisynchronization and $\mathrm{H} \infty$ anti-synchronization for memristive neural networks with mixed time delays and reaction-diffusion terms," Neurocomputing, vol. 168, pp. 726-740, 2015.

[25] D. Zeng, R. Zhang, Y. Liu, and S. Zhong, "Sampled-data synchronization of chaotic Lur'e systems via input-delaydependent-free-matrix zero equality approach," Applied Mathematics and Computation, vol. 315, pp. 34-46, 2017.

[26] S. Ding and Z. Wang, "Stochastic exponential synchronization control of memristive neural networks with multiple timevarying delays," Neurocomputing, vol. 162, pp. 16-25, 2015.

[27] R. Zhang, D. Zeng, S. Zhong, and K. Shi, "Memory feedback PID control for exponential synchronisation of chaotic Lur'e systems," International Journal of Systems Science, vol. 48, no. 12, pp. 2473-2484, 2017.

[28] A. Abdurahman, H. Jiang, and Z. Teng, "Finite-time synchronization for memristor-based neural networks with timevarying delays," Neural Networks, vol. 69, pp. 20-28, 2015.

[29] A. Chandrasekar, R. Rakkiyappan, J. Cao, and S. Lakshmanan, "Synchronization of memristor-based recurrent neural networks with two delay components based on second-order reciprocally convex approach," Neural Networks, vol. 57, pp. 7993, 2014.

[30] W. Wang, L. Li, H. Peng et al., "Anti-synchronization of coupled memristive neutral-type neural networks with mixed timevarying delays via randomly occurring control," Nonlinear Dynamics, vol. 83, no. 4, pp. 2143-2155, 2016.

[31] D. Zeng, R. Zhang, S. Zhong, J. Wang, and K. Shi, "Sampleddata synchronization control for Markovian delayed complex dynamical networks via a novel convex optimization method," Neurocomputing, vol. 266, pp. 606-618, 2017.

[32] X. Luo, J. Deng, J. Liu, W. Wang, X. Ban, and J. Wang, "A quantized kernel least mean square scheme with entropy-guided learning for intelligent data analysis," China Communications, vol. 14, no. 7, pp. 127-136, 2017.
[33] X. Luo, J. Deng, W. Wang, J.-H. Wang, and W. Zhao, "A quantized kernel learning algorithm using a minimum kernel risk-sensitive loss criterion and bilateral gradient technique," Entropy, vol. 19, no. 7, article 365, 2017.

[34] R. Cheng and M. Peng, "Adaptive synchronization for complex networks with probabilistic time-varying delays," Journal of The Franklin Institute, vol. 353, no. 18, pp. 5099-5120, 2016.

[35] G. Nagamani and S. Ramasamy, "Stochastic dissipativity and passivity analysis for discrete-time neural networks with probabilistic time-varying delays in the leakage term," Applied Mathematics and Computation, vol. 289, pp. 237-257, 2016.

[36] C. Pradeep, A. Chandrasekar, R. Murugesu, and R. Rakkiyappan, "Robust stability analysis of stochastic neural networks with Markovian jumping parameters and probabilistic timevarying delays," Complexity, vol. 21, no. 5, pp. 59-72, 2016.

[37] R. Li, J. Cao, and Z. Tu, "Passivity analysis of memristive neural networks with probabilistic time-varying delays," Neurocomputing, vol. 191, pp. 249-262, 2016.

[38] A. Chandrasekar, R. Rakkiyappan, and X. Li, "Effects of bounded and unbounded leakage time-varying delays in memristor-based recurrent neural networks with different memductance functions," Neurocomputing, vol. 202, pp. 67-83, 2016.

[39] G. Nagamani and S. Ramasamy, "Dissipativity and passivity analysis for discrete-time T-S fuzzy stochastic neural networks with leakage time-varying delays based on Abel lemma approach," Journal of The Franklin Institute, vol. 353, no. 14, pp. 3313-3342, 2016.

[40] R. Samidurai and R. Manivannan, "Robust passivity analysis for stochastic impulsive neural networks with leakage and additive time-varying delay components," Applied Mathematics and Computation, vol. 268, Article ID 21387, pp. 743-762, 2015.

[41] L. J. Banu and P. Balasubramaniam, "Robust stability analysis for discrete-time neural networks with time-varying leakage delays and random parameter uncertainties," Neurocomputing, vol. 179, pp. 126-134, 2016.

[42] Y. Tang, R. Qiu, J.-a. Fang, Q. Miao, and M. Xia, "Adaptive lag synchronization in unknown stochastic chaotic neural networks with discrete and distributed time-varying delays," Physics Letters A, vol. 372, no. 24, pp. 4425-4433, 2008.

[43] M. Fattahi and A. Afshar, "Distributed consensus of multi-agent systems with fault in transmission of control input and timevarying delays," Neurocomputing, vol. 189, pp. 11-24, 2016.

[44] Y. Du, S. Zhong, J. Xu, and N. Zhou, "Delay-dependent exponential passivity of uncertain cellular neural networks with discrete and distributed time-varying delays," ISA Transactions, vol. 56, pp. 1-7, 2015.

[45] S. Yang, C. Li, and T. Huang, "Finite-time stabilization of uncertain neural networks with distributed time-varying delays," Neural Computing and Applications, vol. 28, supplement 1, pp. 1155-1163, 2016. 


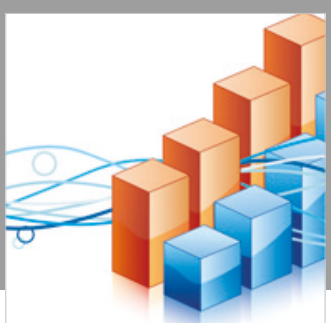

Advances in

Operations Research

\section{-n-m}
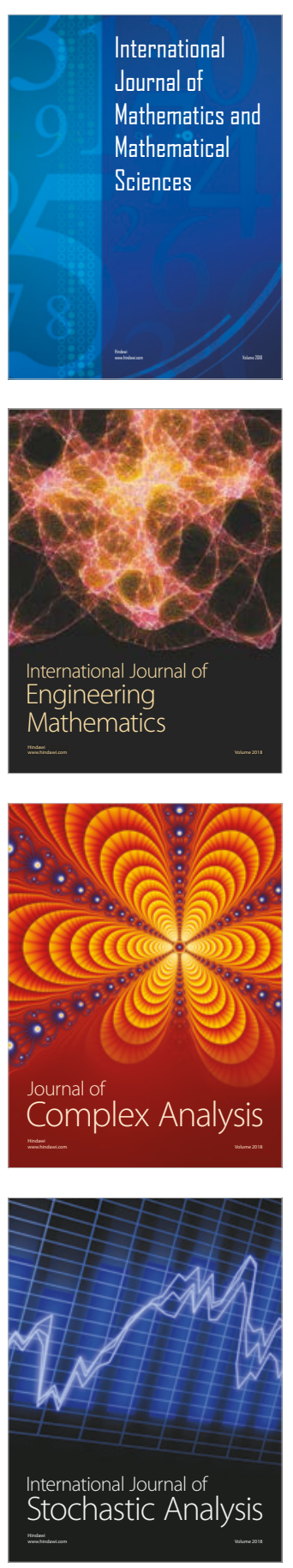
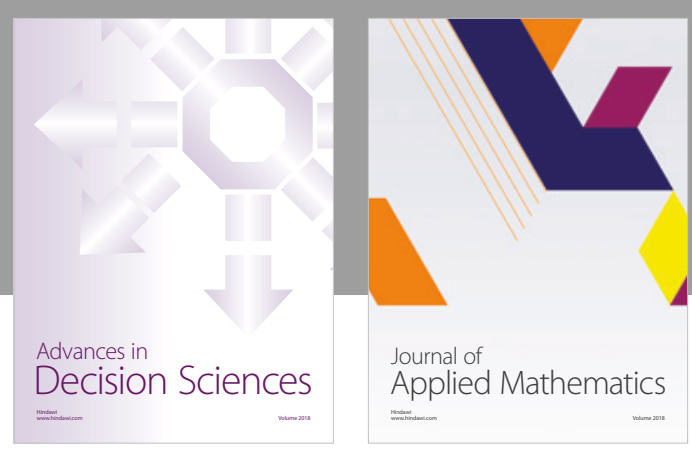

Journal of

Applied Mathematics
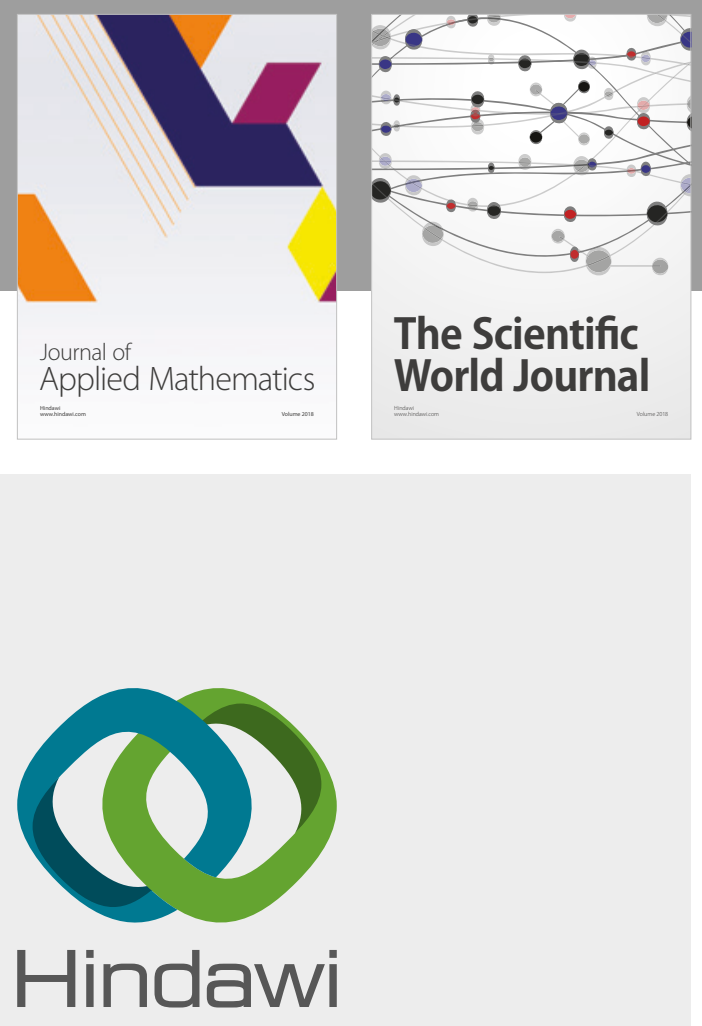

Submit your manuscripts at

www.hindawi.com

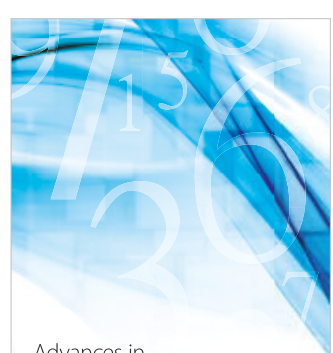

Advances in
Numerical Analysis
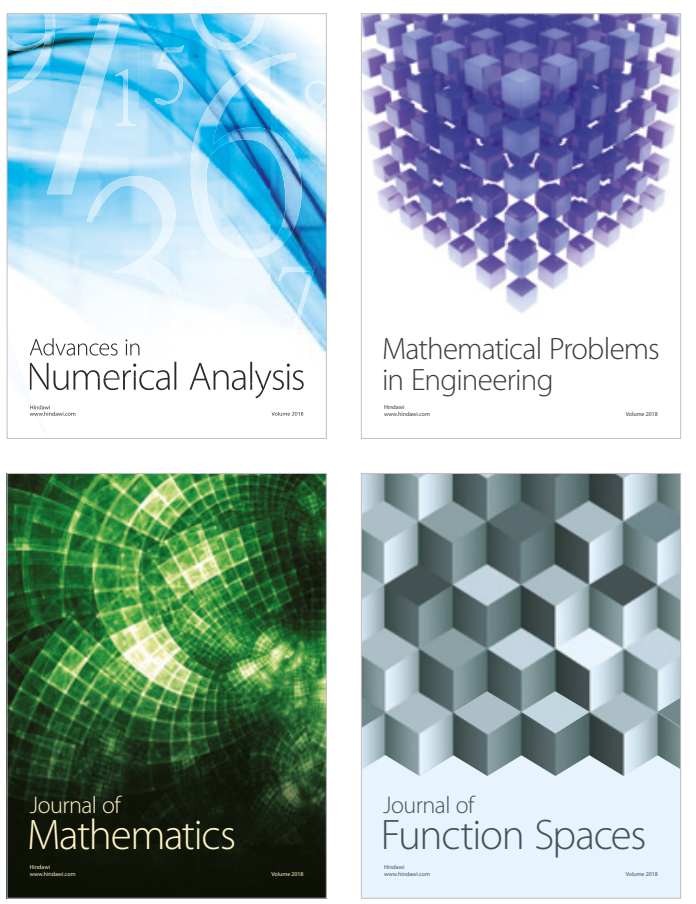

Mathematical Problems in Engineering

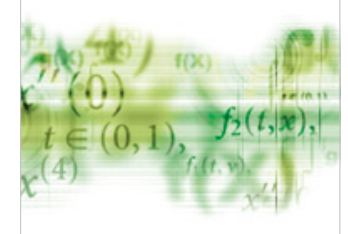

International Journal of

Differential Equations

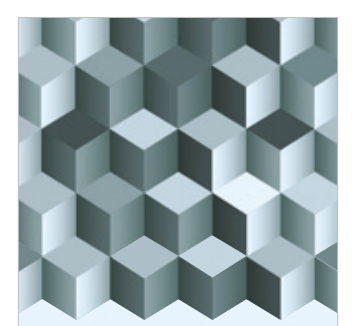

Journal of

Function Spaces

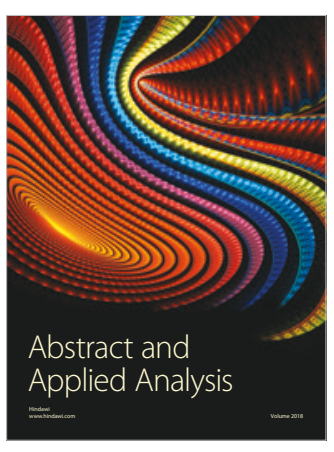

The Scientific

World Journal

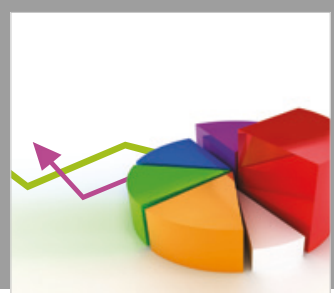

Journal of

Probability and Statistics
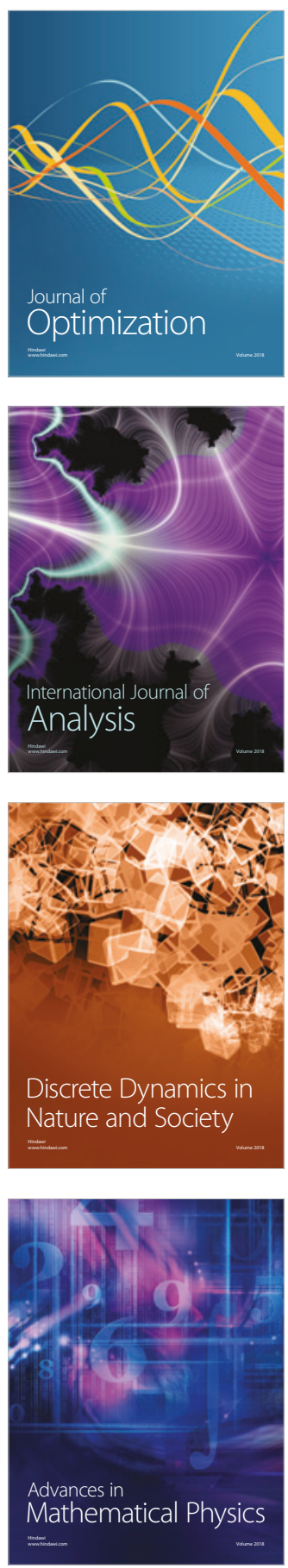